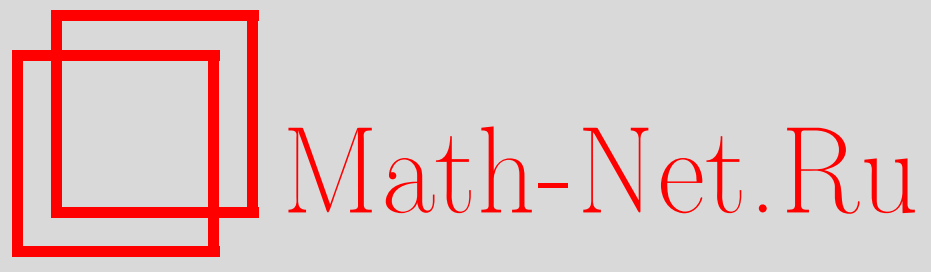

В. И. Юдович, О конвекции сильно вязкой нетеплопроводной жидкости, Матем. сб., 2007, том 198, номер 1, 127-158

DOI: https://doi.org/10.4213/sm1554

Использование Общероссийского математического портала Math-Net.Ru подразумевает, что вы прочитали и согласны с пользовательским соглашением http://www . mathnet.ru/rus/agreement

Параметры загрузки:

IP : 54.164 .48 .24

26 апреля 2023 г., 15:35:43

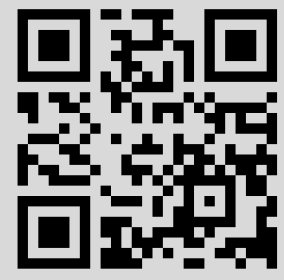


В.И. Юдович

\section{О конвекции сильно вязкой нетеплопроводной жидкости}

Рассматривается асимптотическая модель конвекции Обербека-Буссинеска в случае, когда теплопроводность $\delta=0$, а вязкость $\mu=+\infty$. Доказаны глобальные теоремы существования и единственности решения основной начально-краевой задачи; рассмотрены как классические, так и обобщенные решения. Доказано, что все решения стремятся к некоторым равновесиям при $t \rightarrow \mp \infty$.

Библиография: 41 название.

\section{Введение}

В статье установлено, что в предельном случае нулевой теплопроводности и бесконечно большой вязкости все решения системы Обербека-Буссинеска стремятся к равновесиям при $t \rightarrow+\infty$ и $t \rightarrow-\infty$. Доказаны также глобальные теоремы существования и единственности решения соответствующей начальнокраевой задачи.

Статья состоит из шести параграфов.

В $§ 1$ дана постановка начально-краевой задачи конвекции для случая, когда теплопроводность $\delta=0$, а вязкость $\mu=+\infty$.

В $\S 2$ дается обзор некоторых общих свойств системы: обратимость, инвариантность относительно некоторого действия группы гомотетий, монотонное убывание со временем потенциальной энергии. Как доказано далее (см. §5), последнее свойство влечет затухание движения при $t \rightarrow \mp \infty$. Имеется подпространство фазового пространства системы, на котором в бесконечно далеком прошлом начинаются и в бесконечно далеком будущем заканчиваются все движения. Существенное свойство системы, связанное с отсутствием теплопроводности, - равноизмеримость полей температуры в любые два момента времени для каждого движения. Из этого свойства следует существование большого числа интегралов, а также и принцип максимума-минимума температуры.

В $\S \S 3$ и 4 доказаны глобальные теоремы существования и единственности решения начально-краевой задачи (впрочем, теоремы единственности для задач с начальными условиями всегда локальны). В 33 рассмотрены классические, а в 44 - обобщенные решения. Применяются два метода: надлежащим образом обобщенный метод последовательных приближений Гюнтера-Лихтенштейна и метод исчезающей теплопроводности. Известные трудности вызывает доказательство теоремы единственности обобщенного решения при недифференцируемом поле температур. Для температур класса $L_{3}(D)$, где $D$ - область течения,

Работа выполнена при поддержке Российского фонда фундаментальных исследований (грант № 05-01-00567) и фонда INTAS (грант № 04-80-7297). 
результат достигается копированием рассуждений из [1], касающихся уравнений Эйлера, с использованием оценок потенциала и соответствующей теоремы вложения из [2], [3] в предельном случае теоремы вложения С.Л. Соболева. Хотя глобальную теорему существования удается доказать и в случае $L_{p}(D)$, $p>1$, вопрос о его единственности остается открытым. Ситуация аналогична той, которая имеет место в случае уравнений Эйлера - все известные методы отказывают.

В $\S 5$ показано, что все движения затухают при $t \rightarrow \mp \infty$, причем скорость стремится к нулю по энергетической норме оператора Стокса (т.е. в $\left.W_{2}^{1}(D)\right)$, а температура слабо в $L_{2}(D)$ сходится к некоторым предельным равновесным профилям $\theta_{ \pm}(z)$ соответственно, здесь $z$ - вертикальная координата.

В $\S 6$ сделан ряд дополнительных замечаний и намечено возможное развитие этой работы. В частности, изложена гипотетическая картина турбулентной конвекции при малой теплопроводности и большой вязкости. Работа создает прочную основу для построения соответствующей строгой асимптотической теории.

В настоящей статье в существенно усовершенствованном виде представлены результаты препринта [4].

\section{§1. Постановка задачи. Уравнения ползущих течений}

Рассматривается начально-краевая задача для неизвестных полей температуры $\theta(x, t)$, скорости $\boldsymbol{v}(x, t)$ и давления $P(x, t)$ :

$$
\begin{gathered}
\frac{d \theta}{d t}=0, \\
0=-\nabla P+\Delta \boldsymbol{v}+\theta \boldsymbol{k}, \\
\operatorname{div} \boldsymbol{v}=0,
\end{gathered}
$$

где $d / d t=\partial / \partial t+\boldsymbol{v} \cdot \nabla, \boldsymbol{k}$ - направленный вверх единичный вектор в ограниченной области $D \subset \mathbb{R}^{3}$ (или $\mathbb{R}^{2}$ ) с достаточно гладкой границей $\partial D$ - скажем, класса $C^{2}$. Через $x=\left(x_{1}, x_{2}, x_{3}\right)$ обозначается точка пространства $\mathbb{R}^{3}\left(x_{3}\right.$ будем также называть $z), t$ - время. Граница $\partial D$ предполагается неподвижной, так что на ней выполняется краевое условие

$$
\left.\boldsymbol{v}\right|_{\partial D}=0
$$

В начальный момент $t=0$ задана температура

$$
\left.\theta\right|_{t=0}=f(x)
$$

Система (1.1)-(1.3) описывает медленные конвективные движения неравномерно нагретой жидкости в поле тяжести в предельном случае нулевой теплопроводности и бесконечно большой вязкости. Поясним кратко происхождение этой системы. 
Общие уравнения конвекции Обербека-Буссинеска могут быть записаны в виде (см. [5], [6])

$$
\begin{gathered}
\frac{d \theta}{d t}=\delta \Delta \theta \\
\frac{d \boldsymbol{u}}{d t}=-\nabla P+\mu \Delta \boldsymbol{u}+\theta \boldsymbol{k} \\
\operatorname{div} \boldsymbol{u}=0,
\end{gathered}
$$

где $d / d t=\partial / \partial t+\boldsymbol{u} \cdot \nabla$. При этом на границе $\partial D$ ставятся краевые условия

$$
\left.\boldsymbol{u}\right|_{\partial D}=0,\left.\quad \theta\right|_{\partial D}=-z .
$$

Здесь $\mu$ и $\delta$ - безразмерные коэффициенты вязкости и теплопроводности [5]. $\mathrm{C}$ более часто употребляемыми числами Рэлея $r$, Грасгофа Gr и Прандтля $\sigma$ они связаны соотношениями

$$
r=\frac{1}{\mu \delta}, \quad \mathrm{Gr}=\frac{1}{\mu^{2}}, \quad \sigma=\frac{\mu}{\delta} .
$$

Заметим, что число Прандтля $\sigma$ в отличие от остальных параметров $r, \mathrm{Gr}$, $\mu$ и $\delta$ определяется лишь свойствами жидкости. При этом $\sigma$ может принимать весьма различные по порядку величины значения - порядка $10^{-2}$ для ртути, порядка 1 для воздуха, порядка 10 для воды, порядка $10^{2}-10^{3}$ для минеральных масел, порядка $10^{12}-10^{14}$ для вулканической магмы, порядка $10^{23}$ для вещества мантии Земли. Результаты настоящей статьи, относящиеся к асимптотике больших чисел Прандтля, $\sigma \rightarrow+\infty$, подходят, в особенности, для описания конвекции жидкостей типа магмы, хотя зачастую оказываются применимыми и к обыкновенным жидкостям.

Линейный по $z$ профиль температуры в (1.9) соответствует тому случаю, когда возможно равновесие жидкости $(\boldsymbol{u}=0, \theta=-z)$ при любом $\delta>0$. Знак минус отвечает подогреву жидкости снизу. При нагреве сверху равновесие $\boldsymbol{u}=0, \theta=z$ остается глобально устойчивым при любых $\mu>0, \delta>0$.

Добавляя к уравнениям (1.6)-(1.8) начальные условия

$$
\left.\boldsymbol{u}\right|_{t=0}=\boldsymbol{v}_{0}(x),\left.\quad \theta\right|_{t=0}=f(x)
$$

приходим к общей начально-краевой задаче конвекции Обербека-Буссинеска.

Замена переменных $\boldsymbol{u}=\boldsymbol{v} / \mu, t=\mu \tau$ приводит уравнения (1.6)-(1.7) к виду

$$
\begin{gathered}
\operatorname{Gr} \frac{d \boldsymbol{v}}{d \tau}=-\nabla P+\Delta \boldsymbol{v}+\theta \boldsymbol{k}, \\
\frac{d \theta}{d \tau}=\frac{1}{r} \Delta \theta .
\end{gathered}
$$

В предельном случае, когда $\mathrm{Gr} \rightarrow 0$ и $r \rightarrow \infty$, уравнения (1.11) и (1.12) превращаются в уравнения (1.2) и (1.1) (с заменой $\tau$ на $t$ ). Заметим, что данный предельный переход соответствует случаю, когда вязкость $\mu \rightarrow \infty$, теплопроводность $\delta \rightarrow 0$ и при этом $\mu \delta \rightarrow 0$. 
Уравнение (1.2) уже не содержит производной по времени и имеет смысл связи, скорость "следит" за температурой. Поэтому отпадает начальное условие для скорости.

В уравнении (1.1) нет диффузионного члена. Поэтому отпало и краевое условие для температуры.

Разумеется, построение полной асимптотики начально-краевой задачи для системы (1.10), (1.11) при $\mathrm{Gr} \rightarrow 0, r \rightarrow \infty$ должно еще включать исследование временно́го пограничного слоя для скорости и пограничного слоя вблизи границы $\partial D$ для температуры. Соответствующие построения должны послужить предметом следующих работ.

\section{§ 2. Общие свойства конвекции нетеплопроводной жидкости}

В этом параграфе изучаются некоторые общие свойства (гладких) решений начально-краевой задачи (1.1)-(1.5). Полученные здесь результаты, в частности априорные оценки, будут использованы в 33 при доказательстве глобальной разрешимости, единственности и гладкости решений, а в $55-$ при исследовании предельного поведения решений, когда $t \rightarrow+\infty$ или $t \rightarrow-\infty$.

Из уравнений (1.2)-(1.3) с краевым условием (1.4) можно в каждый момент времени $t$ выразить скорость $\boldsymbol{v}=\boldsymbol{v}(x, t)$ через температуру $\theta$ :

$$
\boldsymbol{v}=G(\theta \boldsymbol{k})=L \theta
$$

где $G$ - оператор Грина системы Стокса, а оператор $L$ определяется этим равенством.

Оператор $G$ заданному векторному полю $\boldsymbol{h}$ ставит в соответствие решение $\boldsymbol{v}=G \boldsymbol{h}$ краевой задачи

$$
\begin{gathered}
0=-\nabla P+\Delta \boldsymbol{v}+\boldsymbol{h}, \\
\operatorname{div} \boldsymbol{v}=0, \\
\left.\boldsymbol{v}\right|_{\partial D}=0 .
\end{gathered}
$$

Эта краевая задача, как в обобщенной, так и в классической постановке, довольно хорошо изучена [7]-[9]. В частности, известно, что при любом $\boldsymbol{h} \in$ $\mathbb{L}_{p}(D), p>1$, решение $\boldsymbol{v}=G \boldsymbol{h} \in W_{p}^{(2)}(D)$ существует и выполняется неравенство коэрцитивности

$$
\|\boldsymbol{v}\|_{W_{p}^{(2)}} \leqslant C_{p}\|\boldsymbol{h}\|_{L_{p}}
$$

с константой $C_{p}$, зависящей лишь от $p$ и от области $D$. Из этой оценки, пользуясь теоремой вложения Соболева, можно вывести оценки для самого поля $\boldsymbol{v}$ и его первых производных $\partial v_{i} / \partial x_{j}$ в $L_{q}$, а также и в пространствах Гёльдера, если $p$ достаточно велико. Заметим лишь, что можно эти последние оценки получить при несколько меньших требованиях к гладкости границы.

Пространство векторных полей $\mathbb{L}_{p}$ допускает прямое разложение $\mathbb{L}_{p}=S_{p}+G_{p}$ при любом $p>1$, где $S_{p}$ - замыкание множества $M$ (множества $C^{\infty}$-гладких соленоидальных финитных в $D$ векторных полей) по норме $\mathbb{L}_{p}$, а $G_{p}$ - замыкание по той же норме множества градиентов всевозможных гладких в $D$ функций 
(см. [9; гл. $1, \S 5])$. В гидродинамике фундаментальную роль играет проектор $\Pi: \mathbb{L}_{p} \rightarrow S_{p}$, соответствующий данному прямому разложению. В случае $p=2$ это ортогональный проектор.

Заметим, что краевую задачу (2.2) можно представить как операторное уравнение $-\Pi \cdot \Delta \boldsymbol{v}=\Pi \boldsymbol{h}$. Оператор $-\Pi \cdot \Delta$ с областью определения, состоящей из соленоидальных полей класса $W_{p}^{(2)}$, исчезающих на границе $\partial D$, называется оператором Стокса. В случае $p=2$ он самосопряжен и положительно определен. Его энергетическое пространство $H_{1}$ есть замыкание множества $M$ по норме, отвечающей скалярному произведению

$$
(\boldsymbol{v}, \boldsymbol{w})_{H_{1}}=\int_{D} \nabla \boldsymbol{v} \cdot \nabla \boldsymbol{w} d x=\int_{D} \frac{\partial v_{i}}{\partial x_{j}} \frac{\partial w_{i}}{\partial x_{j}} d x
$$

Пространство $H_{1}$ естественно вложено в $W_{2}^{(1)}(D)$ в случае ограниченной области $D$. При этом существование обобщенных производных $\partial v_{i} / \partial x_{j}$ имеет место, конечно, для любой области, а принадлежность поля $\boldsymbol{v}$ пространству $\mathbb{L}_{2}$ получается при помощи неравенства Фридрихса, которое справедливо и для некоторых неограниченных областей, скажем для цилиндра или слоя.

Подстановка (2.1) в (1.1) приводит задачу к одному обыкновенному дифференциальному уравнению в банаховом пространстве (норму уточним позднее) функций $\theta$ :

$$
\dot{\theta}=K(L \theta, \theta) .
$$

Здесь $K-$ билинейный оператор на $S_{2} \times L_{2}$ и $K(\boldsymbol{v}, \theta)=-\boldsymbol{v} \cdot \nabla \theta=-\operatorname{div}(\theta \boldsymbol{v})$. Он обладает гироскопическим свойством: для любых гладких поля $\boldsymbol{v} \in S_{2}$ и функций $\theta, \varphi$ выполнено равенство

$$
(K(\boldsymbol{v}, \theta), \varphi)=-(K(\boldsymbol{v}, \varphi), \theta),
$$

где $(\cdot, \cdot)$ - скалярное произведение в $L_{2}(D)$. В частности, $(K(\boldsymbol{v}, \theta), \theta)=0$.

ПРЕДЛОЖениЕ 2.1. Система (1.1)-(1.2) обратима: она инвариантна относительно замены $t \mapsto-t, \theta \mapsto-\theta, \boldsymbol{v} \mapsto-\boldsymbol{v}$. Она инвариантна также и относительно замены $\gamma t \mapsto t, \theta \mapsto \gamma \theta, \boldsymbol{v} \mapsto \gamma \boldsymbol{v}, P \rightarrow \gamma P$ при любом вещественном $\gamma \neq 0$, так что, вместе с $\theta(x, t), \boldsymbol{v}(x, t), P(x, t)$, также и $\gamma \theta(x, \gamma t)$, $\gamma \boldsymbol{v}(x, \gamma t), \gamma P(x, \gamma t)$ есть решение.

Доказательство очевидно. Напомним, что вообще уравнение

$$
\dot{\theta}=F(\theta)
$$

в банаховом пространстве $X$ называется обратимым [10]-[14], если оно инвариантно относительно замены $t \mapsto-t, \theta \mapsto J \theta$, где $J: X \rightarrow X-$ инверсия $\left(J^{2}=I\right.$, $J \neq I)$. Условие обратимости с инверсией $J$ имеет вид

$$
F(J \theta)=-J^{\prime}(\theta) F(\theta)
$$

для всех $\theta \in X$. Если $J$ - линейный оператор, то это условие упрощается и принимает вид $F J=-J F$. В случае уравнения $(2.3) \quad J=-I$. 
ПреДЛОЖенИЕ 2.2. Для любой (пусть гладкой) функиии $F: \mathbb{R} \rightarrow \mathbb{R}$ функицонал

$$
\theta \rightarrow \int_{D} F(\theta) d x
$$

есть интеграл системы (1.1)-(1.2).

Доказательство сразу следует из равенств, справедливых для любого решения системы (1.1)-(1.4):

$$
\frac{d}{d t} \int_{D} F(\theta) d x=\int_{D}-F^{\prime}(\theta) \boldsymbol{v} \cdot \nabla \theta d x=-\int_{D} \boldsymbol{v} \cdot \nabla F(\theta) d x=-\int_{\partial D} \boldsymbol{v}_{n} F(\theta) d S=0 .
$$

Определим потенциальную энергию $V[\theta]$ и потенциал $\Phi[\theta]=-V(\theta)$ жидкости в поле тяжести, полагая

$$
V[\theta]=-\int_{D} \theta z d x
$$

ПРедложениЕ 2.3. Потенциальная энергия для любого движения монотонно убывает со временем (а потенциал возрастает).

ДокАзАТЕльство. Из (1.1)-(1.4) следует равенство

$$
\frac{d}{d t} \int_{D} \theta z d x=\int_{D}(\nabla \boldsymbol{v})^{2} d x
$$

Заметим, что правая часть обращается в нуль только при $v=0$.

ПРЕДЛОЖЕНИЕ 2.4. Всякое стационарное решение $\boldsymbol{v}_{0}, \theta_{0}$ системы (1.1)(1.2) есть равновесие: $\boldsymbol{v}_{0}=0, a \theta_{0}=\theta_{0}(z)$ локально, в окрестности каждой точки $x \in D$; при этом $\theta_{0}$ - произвольная гладкая функиия.

ДокАЗАТЕЛЬство. Из (2.4) следует, что $\boldsymbol{v}_{0}=0$. А система (1.1)-(1.2) принимает тогда вид

$$
\frac{\partial p}{\partial x_{1}}=0, \quad \frac{\partial p}{\partial x_{2}}=0, \quad \frac{\partial p}{\partial x_{3}}=\theta .
$$

Из первых двух уравнений (2.5) следует, что $p$, по крайней мере локально, есть функция одной лишь координаты $x_{3}=z$. Тогда то же свойство функции $\theta$ следует из третьего уравнения (2.5), что и требовалось доказать.

Может показаться удивительным, но система (2.5) для некоторых областей $D$ может иметь решения, зависящие от $x_{1}, x_{2}$.

Введем следующие обозначения. Для любых вещественных $z_{1}<z_{2}$ обозначим через $D_{z_{1}, z_{2}}$ пересечение области $D$ и слоя, определяемого неравенствами $z_{1}<x_{3}<z_{2}$. Через $S_{z}$ обозначим сечение области $D$ горизонтальной плоскостью $x_{3}=z$. Таким образом,

$$
D_{z_{1}, z_{2}}=\left\{x \mid x \in D, z_{1}<x_{3}<z_{2}\right\}, \quad S_{z}=\left\{x \mid x \in D, x_{3}=z\right\} .
$$

Горизонтальные плоскости появляются здесь в качестве эквипотенциальных поверхностей силы тяжести. 
ПРЕДЛОЖЕНИЕ 2.5. Для того чтобъ всякое $C^{\infty}$-гладкое решение $p=$ $p\left(x_{1}, x_{2}, x_{3}\right)$ системь уравнений

$$
\frac{\partial p}{\partial x_{1}}=0, \quad \frac{\partial p}{\partial x_{2}}=0
$$

в области $D \subset \mathbb{R}^{3}$ было функцией $p=p\left(x_{3}\right)$, необходимо и достаточно, чтобъ для любых $z_{1}<z_{2}$ открытое множество $D_{z_{1}, z_{2}}$ было связно (оно может быть u пустым).

ДокАЗАТЕЛЬСтво. Если условие выполнено, то связными являются также сечения $S_{z}$ при любых $z$. Рассмотрим гладкую кривую

$$
z \mapsto \bar{x}(z)=\left(\bar{x}_{1}(z), \bar{x}_{2}(z), \bar{x}_{3}(z)\right),
$$

определенную для всех $z$ из некоторого интервала $\mathscr{J}$, содержащего всевозможные значения вертикальной переменной $x_{3}$ в области $D$. Потребуем, чтобы для всех $x \in \mathscr{J}$ функция $\bar{x}_{3}(z)$ строго монотонно возрастала. Такая кривая (гладкое сечение слоения области $D$ на горизонтальные сечения) всегда существует.

Если сечение $S_{z}$ для данного $z \in \mathscr{J}$ связно, то всякое решение системы (2.6) постоянно на $S_{z}$. Поэтому

$$
p\left(x_{1}, x_{2}, z\right)=p\left(\bar{x}_{1}(z), \bar{x}_{2}(z), z\right) .
$$

Если все сечения связны, то равенство (2.7) выполняется всюду в $D$, что доказывает предложение 2.5 в части достаточности.

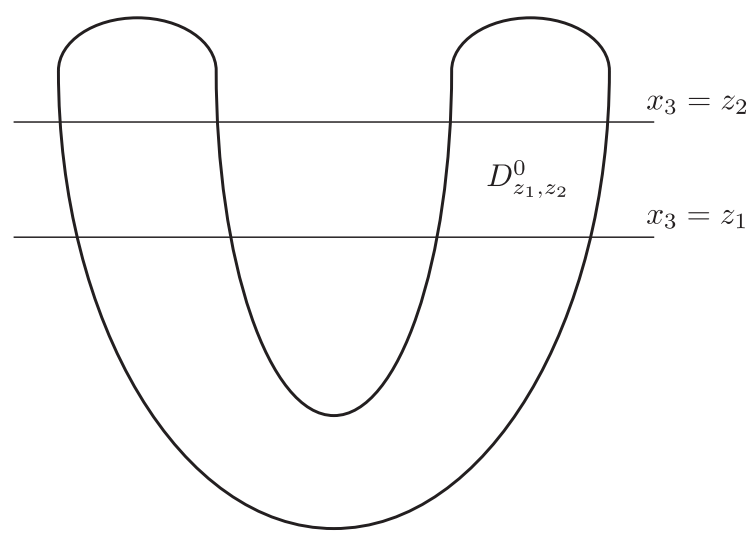

Рис. 1

Теперь предположим, что при некоторых $z_{1}$ и $z_{2}$ множество $D_{z_{1}, z_{2}}$ несвязно. Пусть $D_{z_{1}, z_{2}}^{0}$ - одна из его компонент связности (см. рис. 1). Определим функцию $p\left(x_{1}, x_{2}, x_{3}\right)$, полагая

$$
p\left(x_{1}, x_{2}, x_{3}\right)= \begin{cases}p_{0}(z), & \left(x_{1}, x_{2}, x_{3}\right) \in D_{z_{1}, z_{2}}^{0}, \\ 0, & \left(x_{1}, x_{2}, x_{3}\right) \in D \backslash D_{z_{1}, z_{2}}^{0} .\end{cases}
$$


Здесь $p_{0}(z)-C^{\infty}$-гладкая финитная на интервале $\left(z_{1}, z_{2}\right)$ функция. Очевидно, так определенная функция $p$ является $C^{\infty}$-гладкой в $D$. Наличие решения (2.8), зависящего от $x_{1}$ и $x_{2}$, доказывает необходимость условия предложения 2.5. Что и требовалось доказать.

Приведенное доказательство разъясняет причину появления у системы (2.6) решений, зависящих от $x_{1}, x_{2}$ - см. (2.8). Локально всякое решение системы (2.6), конечно, есть $p(z)$. Если, однако, область $D$ имеет $U$-образную форму (или, скажем, $W$-образна) и содержит несколько "рукавов", то в каждом из них может быть свой профиль $p(z)$. Нужно лишь позаботиться о выполнении условий сопряжения этих профилей на критических уровнях, там, где соединяются рукава.

Если рассматривать аналитические решения, то никакой проблемы не возникает, ибо локально определенный аналитический профиль $p(z)$ однозначно продолжается на всю область $D$. Это назидательный пример ситуации, когда математический нюанс - различие между $C^{\omega}$ и $C^{\infty}$ - имеет ясный и "грубый" физический смысл.

Заметим еще, что выбор класса $C^{\infty}$ в предложении 2.5 довольно случаен. Утверждение остается в силе, если, например, рассматривать решения класса $C^{1}$, либо кусочно гладкие решения, либо решения класса $W_{q}^{(\ell)}$ и т.п.

Когда нет теплопроводности, равновесным оказывается произвольный профиль температуры. При наличии теплопроводности вырабатывается линейный по $z$ профиль. Интересно, что линейные по $z$ профили сохраняют некоторую особую роль и для модели (1.1)-(1.2).

ПрЕДЛОЖЕНИЕ 2.6. Любое движение $\theta(t)=\theta(\cdot, t)$ со временем приближается в метрике $L_{2}(D)$ ко всем линейным устойчивым профилям $C z, C>0$, и удаляется от всех неустойчивых профилей $C z, C<0$.

ДокАзАТЕльство. Так как согласно предложению $2.2\|\theta(t)\|_{L_{2}}$ постоянна, используя (2.4), выводим

$$
\frac{1}{2} \frac{d}{d t} \int_{D}(\theta+C z)^{2} d x=C \int_{D}(\nabla \boldsymbol{v})^{2} d x
$$

с произвольной постоянной $C$.

В частности,

$$
\frac{1}{2} \frac{d}{d t} \int_{D}(\theta \mp z)^{2} d x=\mp \int_{D}(\nabla \boldsymbol{v})^{2} d x .
$$

ПРЕДЛОЖЕНИЕ 2.7. Для любого движения существуют предель

$$
\lim _{t \rightarrow \mp \infty} V[\theta(t)]=V_{\mp}
$$

и выполнено равенство

$$
V_{-}-V_{+}=\int_{-\infty}^{\infty} \int_{D}(\nabla \boldsymbol{v})^{2} d x d t
$$


ДокАЗАТЕЛЬство. Для любого движения потенциальная энергия $V[\theta(t)]-$ монотонно убывающая функция времени (предложение 2.3), притом ограниченная - это следует из предложения 2.2. Поэтому существуют пределы (2.9), а интегрирование равенства (2.4) дает (2.10), что и требовалось доказать.

Дальше показано (см. лемму 5.5), что для всякого движения температура $\theta(t)$ слабо сходится в $L_{p}$ при $t \rightarrow \pm \infty$ соответственно к $\theta_{+}$и $\theta_{-}$, где $\theta_{+}$и $\theta_{-}-$ равновесные распределения температуры. Так как $V$ - слабо непрерывный функционал на $L_{p}$ (при любом $p>1$ ), имеем равенство

$$
\lim _{t \rightarrow \mp \infty} V[\theta(t)]=V_{\mp}=V\left(\theta_{ \pm}\right) .
$$

Смысл важного равенства (2.10) ясен: полная потеря потенииальной энергии на траектории равна энергии, диссипированной вязкостъю.

Естественно возникает следующая гипотеза.

ПреДЛОжЕНИЕ А. Всякое движение при $t \rightarrow \mp \infty$ стремится $\kappa$ равновесию: скорость $v$ стремится $\kappa$ нулю, а температура $-\kappa$ равновесным распределениям $\theta_{\mp}=\theta_{\mp}(z)$.

Это утверждение оставим пока без доказательства и без строгой формулировки, поскольку не сказано, в каком смысле понимается сходимость (см. §5).

ПредложениЕ 2.8 (принцип максимума). Температура достигает максимума (и минимума) в начальный момент времени.

ДокАзАТЕЛьство получается предельным переходом при $p \rightarrow+\infty$ в равенстве

$$
\|\theta(\cdot, t)\|_{L_{p}(D)}=\|\theta(\cdot)\|_{L_{p}(D)},
$$

вытекающем из предложения 2.2 .

В действительности верно нечто большее. Рассмотрим задачу Коши

$$
\dot{x}=\boldsymbol{v}(x, t), \quad x(0)=a \in \bar{D},
$$

определяющую движения жидких частиц. Поскольку поле $\boldsymbol{v}$ соленоидально и касательно к границе $\partial D$, преобразования семейства $g(t): a \mapsto x(a, t)$, где $x(a, t)$ - решение задачи (2.12), сохраняют меру Лебега. Из (1.1) следует, что

$$
\theta(x, t)=f\left(g^{-1}(t) x\right) .
$$

Ввиду компактности $D$ и гладкости $\partial D$ семейство $g(t)$ определено глобально для $t \in \mathbb{R}$.

По любой измеримой функции $f$ на $D$ определим класс $\Lambda_{f}$, к которому отнесем функции вида $f(g x)$, где $g: D \rightarrow D$ сохраняет элемент объема. Две функции $f, h$ класса $\Lambda_{f}$ равноизмеримы (см. [15]-[18]): для любого $s \in \mathbb{R}$ их лебеговы множества бо́льших значений имеют одинаковые меры

$$
\operatorname{mes}\{x: x \in D, f(x)>s\}=\operatorname{mes}\{x: x \in D, h(x)>s\} \text {. }
$$


ПРЕДЛОЖЕНИЕ 2.9. Класс $\Lambda_{f}$ инвариантен относительно движений системы (1.1)-(1.2) (уравнения (2.3)) для любой гладкой функиии $f$.

ДоказАТЕЛЬство. См. (2.13).

Из предложения 2.9 следуют, разумеется, предложения 2.2 и 2.8 .

Равноизмеримость (2.14) есть отношение эквивалентности, и пространство измеримых функций расслаивается на листы $\Lambda_{f}-$ классы функций, равноизмеримых с данной функцией $f$. Из равноизмеримости функций $f$ и $h$ можно, используя некоторые результаты теории меры [19], [20], вывести, что $f(x)=$ $h(g x)$, где $g: D \rightarrow D$ сохраняет меру. Правда, может оказаться, что отображение $g$ не взаимно однозначно [21]. Если $f \in L_{1}(D)$, то и все функции листа $\Lambda_{f}$ тоже принадлежат $L_{1}(D)$. То же верно и для $L_{p}(D), 0<p \leqslant \infty$, и для других функциональных пространств с нормами или метриками, инвариантными относительно преобразований, сохраняющих меру (пространства Орлича, Марцинкевича, Гальперина, Лоренца и т.д.). Интересно, конечно, рассматривать и классы равноизмеримых функций в других функциональных пространствах, при этом приходится налагать соответствующие ограничения на отображения $g$.

\section{§ 3. Глобальная разрешимость и единственность}

В этом и следующем параграфах доказаны теоремы существования и единственности решения начально-краевой задачи (1.1)-(1.2) на всей числовой оси времени. В $\S 5$ установлено, что при $t \rightarrow \pm \infty$ каждое решение стремится в определенном смысле к равновесию.

Начально-краевая задача (1.1)-(1.2) попадает в класс уравнений распространения примеси движущейся жидкостью в условиях, когда поле скоростей в каждый момент времени определяется распределением концентраций примеси в тот же момент. Этот класс содержит также уравнения Эйлера для несжимаемой жидкости. При этом роль примеси играет вихрь, так что в трехмерном случае "примесь" векторная.

Давно апробированный и теорией, и вычислительной практикой метод последовательных приближений Гюнтера-Лихтенштейна распространяется на весь указанный класс уравнений. Для рассматриваемой здесь системы имеют место значительные упрощения и улучшения результатов по сравнению с системой уравнений Эйлера. Это связано с лучшими сглаживающими свойствами оператора управления, каковой здесь выражается через оператор Грина системы Стокса.

Сначала будут доказаны теоремы существования и единственности классических решений при минимальных требованиях к заданному начальному полю, которое должно быть непрерывно дифференцируемо. Эти результаты следуют из установленной здесь глобальной сходимости метода последовательных приближений.

Существование обобщенного решения можно получить при помощи того же метода последовательных приближений и априорных оценок. Другой метод, 
примененный ниже, состоит во введении исчезающей теплопроводности и тоже восходит к теории уравнений Эйлера [1]. После предельного перехода нужное интегральное тождество получается первоначально лишь для пробных функций, исчезающих на границе пространственно-временного цилиндра. Доказанная в $§ 4$ лемма 4.1 позволяет избавиться от этого излишнего ограничения.

Некоторые трудности вызывает вопрос о единственности обобщенного решения. Прямолинейное применение энергетического метода, вполне достаточное для классических решений, заставляет требовать от решения дифференцируемости. В то же время естественная область действия обоих применяемых методов определяется условием существования и единственности решения задачи Коши, определяющей движение жидких частиц. Для этого достаточно, чтобы поле скоростей было непрерывно и удовлетворяло условию Липшица или хотя бы условию Осгуда. Согласно известным свойствам оператора управления если поле температур - класса $L_{p}(D), p>3$, то поле скоростей даже непрерывно дифференцируемо, а если оно из $L_{3}(D)$, то, как устанавливается при помощи результатов из [2] (см. также [3]), как раз выполняется условие Осгуда.

Несколько парадоксально, что теорему единственности в этом классе удается доказать лишь копируя рассуждения из [1] - при этом температура уподобляется вихрю, по которому вводится некая фиктивная функция тока. Существование обобщенного решения возможно установить и в более общих классах, чем это здесь сделано - в $L_{p}(D)$ при $p<3$, и даже в некоторых классах обобщенных функций [22], однако как в подобных классах доказывать единственность, неясно. Границу действия известных методов обозначает здесь возможность (или невозможность) определить по полю скоростей движение частиц. Для негладких полей скорости таких, какие возникают в качестве обобщенных решений уравнений гидродинамики, было бы очень интересно научиться определять движения если не отдельных частиц, то хотя бы положительных масс жидкости - скажем, в духе теории транспортного уравнения [23]. Совершенно неясно, однако, как для таких решений доказывать теорему единственности.

Гладкие решения. Метод последовательных приближений. Речь пойдет о глобальной разрешимости и единственности решения начально-краевой задачи (1.1)-(1.5). Как уже отмечалось (см. (1.3) и (1.4)), скорость выражается через температуру: $\boldsymbol{v}=L \theta=G(\theta \boldsymbol{k})$, после чего (1.1) принимает вид

$$
\dot{\theta}=K(L \theta, \theta), \quad K(\boldsymbol{v}, \theta)=-\boldsymbol{v} \cdot \nabla \theta .
$$

Это - уравнение переноса примеси ( $\theta$ играет роль концентрации) движением жидкости с управляемым самой примесью полем скоростей, $L$ - оператор управления. Такую же структуру имеет уравнение Гельмгольца для вихря в двумерных течениях (в трехмерном случае "примесь" векторная), оператор управления $L_{1}$ в этом случае восстанавливает поле скоростей по заданному вихрю (нюансы, связанные с многосвязными областями, сейчас несущественны). Ясно поэтому, что методы, развитые для уравнений Эйлера в случае несжимаемой жидкости, пригодны и для рассматриваемой модели. При этом возникают 
упрощения и усиления, связанные с лучшими сглаживающими свойствами оператора $L$ : он увеличивает дифференцируемость на две единицы, а $L_{1}-$ лишь на единицу. Упрощает, конечно, дело и то, что, в отличие от вихря, $\theta$ - скалярное поле независимо от размерности области течения.

Решение задачи (1.1)-(1.2) можно построить методом последовательных приближений типа Гюнтера-Лихтенштейна [24], [25; с. 482-493], который здесь принимает следующую форму. В качестве начального приближения возьмем для определенности $\theta_{0}=f, \boldsymbol{v}_{0}=L f$. Если в $n$-м приближении поля $\theta_{n}$, $\boldsymbol{v}_{n}=L \theta_{n}$ уже известны, то для определения $\theta_{n+1}, \boldsymbol{v}_{n+1}$ рассматриваем сначала задачу Коши

$$
\dot{x}=v_{n}(x, t), \quad x(0)=a, \quad a \in D .
$$

Пусть $g_{n}(t)$ - ее эволюционный оператор, так что $x(t)=g_{n}(t) a$. Тогда полагаем

$$
\begin{gathered}
\theta_{n+1}(x, t)=f\left(h_{n}(t) x\right), \\
\boldsymbol{v}_{n+1}=L \theta_{n+1}, \quad h_{n}=g_{n}^{-1} .
\end{gathered}
$$

Метод пригоден и для обобщенных решений, но сейчас займемся гладкими.

В следующей теореме требования к начальному полю температуры минимально возможные. Результат о единственности далее будет усилен.

Теорема 3.1. Пусть $D$ - ограниченная область в $\mathbb{R}^{3}$ с границей $\partial D$ класса $C^{2, \gamma}$ для некоторого $\gamma, 0<\gamma \leqslant 1$, а начальная температура $f \in C^{1}(D)$. Тогда существует и притом единственное классическое решение $(\theta, \boldsymbol{v})$ задачи (1.1), (1.2), определенное для всех $t \in \mathbb{R}$.

ДокАЗАтЕльство. Единственность доказывается стандартно. Если $\left(\theta^{\prime}, \boldsymbol{v}^{\prime}\right)$ и $\left(\theta^{\prime \prime}, \boldsymbol{v}^{\prime \prime}\right)$ - классические решения, образуем их разность $(\varphi, \boldsymbol{u})$, где $\varphi=\theta^{\prime}-\theta^{\prime \prime}$, $\boldsymbol{u}=\boldsymbol{v}^{\prime}-\boldsymbol{v}^{\prime \prime}$. Из уравнения для $\varphi$, получаемого вычитанием уравнений, записанных для двух решений уравнения (1.1), умножая на $\varphi$ и интегрируя по области $D$, выводим равенство

$$
\frac{1}{2} \frac{d}{d t} \int_{D} \varphi^{2}(x, t) d x=-\int_{D} u_{j} \frac{\partial \theta^{\prime}}{\partial x_{j}} \varphi d x .
$$

Для $\boldsymbol{u}=L \varphi$, применяя неравенство коэрцитивности и теорему вложения, получаем оценку

$$
\|\boldsymbol{u}\|_{L_{2}(D)} \leqslant C\|\varphi\|_{L_{2}(D)}
$$

с постоянной $C$, зависящей лишь от области $D$.

Кроме того, для любого $T>0$ существует

$$
\max _{(x, t) \in Q_{T}}\left|\nabla \theta^{\prime}(x, t)\right| \leqslant M_{T}, \quad Q_{T}=\bar{D} \times[0, T] .
$$

Теперь из (3.3)-(3.5) вытекает справедливость неравенства

$$
\frac{1}{2} \frac{d}{d t} \int_{D} \varphi^{2}(x, t) d x \leqslant C M_{T} \int_{D} \varphi^{2}(x, t) d x, \quad 0 \leqslant t \leqslant T .
$$


Отсюда следует, что $\varphi(x, t)=0$ для всех $(x, t) \in Q_{T}$, а ввиду произвольности $T-$ и для всех $(x, t) \in D \times \mathbb{R}_{+}$. Для отрицательных времен доказательство такое же. Раз $\varphi=0$, значит, и $\boldsymbol{u}=L \varphi=0$. Единственность, таким образом, доказана.

Существование решения установим, доказав, что последовательные приближения, описанные выше, сходятся. Докажем, что последовательность $\theta_{0}, \theta_{1}, \ldots$, $\theta_{n}, \ldots$ сходится равномерно во всяком замкнутом цилиндре $Q_{T}=\bar{D} \times[0, T]$, $t>0$. Иными словами, убедимся, что ряд

$$
\theta=\theta_{0}+\sum_{n=0}^{\infty}\left(\theta_{n+1}-\theta_{n}\right)
$$

сходится на $Q_{T}$ равномерно.

Из (3.2) следует оценка

$\left|\theta_{n+1}(x, t)-\theta_{n}(x, t)\right| \leqslant M_{f}\left|h_{n}(t) x-h_{n-1}(t) x\right|, \quad M_{f}=\max _{x \in \bar{D}}|\nabla f(x)|, \quad 0 \leqslant t \leqslant T$,

для любых $(x, t) \in Q_{T}$.

Введем обозначения:

$$
\begin{aligned}
& \zeta_{n}(t)=\max _{x \in \bar{D}}\left|\theta_{n+1}(x, t)-\theta_{n}(x, t)\right|, \\
& \eta_{n}(t)=\max _{x \in \bar{D}}\left|h_{n}(t) x-h_{n-1}(t) x\right| .
\end{aligned}
$$

Из (3.7) получаем неравенство

$$
\zeta_{n}(t) \leqslant M_{f} \eta_{n}(t), \quad t \geqslant 0, \quad n=0,1, \ldots
$$

При этом по определению полагаем, что $h_{-1}=\mathrm{id}$.

Глобальный результат о сходимости последовательных приближений оказывается достижимым благодаря наличию в нашем распоряжении сильных равномерных по $n$ оценок. Именно, нормы в $L_{p}(D), 1 \leqslant p \leqslant \infty$, функций $\theta_{n}$ все равны $\|f\|_{L_{p}(D)}$ и не зависят от $t$ и от $n$. Тогда, применяя неравенство коэрцитивности в $L_{p}(D)$ и теорему вложения $W_{p}^{(2)}(D)$ в $C^{1, \lambda}, \lambda=1-3 / p$ при $p>3$, приходим к оценке для $\boldsymbol{v}_{n}=L \theta_{n}$

$$
\left|\nabla \boldsymbol{v}_{n}(x, t)\right| \leqslant M_{v}
$$

равномерной по $x \in \bar{D}, t \in \mathbb{R}, n=0,1, \ldots$. Постоянная $M_{v}$ зависит лишь от $f$ и от $D$.

Чтобы оценить $\eta_{n}(t)$, рассмотрим задачу Коши для системы уравнений движения жидких частиц:

$$
\frac{d x_{n}}{d s}=\boldsymbol{v}_{n}\left(x_{n}, s\right),\left.\quad x_{n}\right|_{s=t}=x .
$$

Тогда $h_{n}(t) x=x(0)$.

Полагая $x_{n}-x_{n-1}=z_{n}=z_{n}(x, t, s)$, из (3.9) выводим:

$$
\frac{d z_{n}}{d s}=\boldsymbol{v}_{n}\left(x_{n}, s\right)-\boldsymbol{v}_{n-1}\left(x_{n-1}, s\right),\left.\quad z_{n}\right|_{s=t}=0 .
$$


Разность полей в (3.10) преобразуем к виду

$$
\left[\boldsymbol{v}_{n}\left(x_{n}, s\right)-\boldsymbol{v}_{n}\left(x_{n-1}, s\right)\right]+\left[\boldsymbol{v}_{n}\left(x_{n-1}, s\right)-\boldsymbol{v}_{n-1}\left(x_{n-1}, s\right)\right] .
$$

Для этих слагаемых имеем оценки

$$
\begin{gathered}
\left|\boldsymbol{v}_{n}\left(x_{n}, s\right)-\boldsymbol{v}_{n}\left(x_{n-1}, s\right)\right| \leqslant M_{v}\left|z_{n}(x, t, s)\right| \\
\left|\boldsymbol{v}_{n}\left(x_{n-1}, s\right)-\boldsymbol{v}_{n-1}\left(x_{n-1}, s\right)\right| \leqslant M_{L} \zeta_{n-1} .
\end{gathered}
$$

Вторая оценка следует из представления $\boldsymbol{v}_{n}-\boldsymbol{v}_{n-1}=L\left(\theta_{n}-\theta_{n-1}\right)$, и константа $M_{L}$, зависящая лишь от $D$, есть соответствующая норма (из $C$ в $C$ ) оператора $L$.

Далее положим

$$
\chi_{n}(s, t)=\max _{x \in \bar{D}}\left|z_{n}(x, t, s)\right| .
$$

Интегрируя (3.10) по $s$, получаем равенство

$$
-z_{n}(x, t, s)=\int_{s}^{t}\left[\boldsymbol{v}_{n}\left(x_{n}(\sigma), \sigma\right)-\boldsymbol{v}_{n-1}\left(x_{n-1}(\sigma), \sigma\right)\right] d \sigma .
$$

Из (3.11)-(3.13) выводим неравенство

$$
\chi_{n}(s, t) \leqslant \int_{s}^{t}\left[M_{v} \chi_{n}(\sigma, t)+M_{L} \zeta_{n-1}(\sigma)\right] d \sigma .
$$

Полагая здесь $s=0$ и замечая, что $\chi_{n}(0, t)=\eta_{n}(t)$, получаем неравенство

$$
\eta_{n}(t) \leqslant \int_{0}^{t}\left[M_{v} \chi_{n}(\sigma, t)+M_{L} \zeta_{n-1}(\sigma)\right] d \sigma .
$$

Из (3.14), фиксируя $t$ и вводя обозначение

$$
q_{n}(s)=\int_{s}^{t} \chi_{n}(\sigma, t) d \sigma
$$

получаем дифференциальное неравенство для $q_{n}$, из которого стандартным образом выводим оценку

$$
q_{n}(s) \leqslant M_{L} \int_{s}^{t} e^{-(s-\sigma) M_{v}} d \sigma \int_{\sigma}^{t} \zeta_{n-1}(\tau) d \tau .
$$

Теперь из (3.15)-(3.17) и (3.8) следует неравенство

$$
\eta_{n}(t) \leqslant M_{v} M_{L} M_{f} \int_{0}^{t} e^{\sigma M_{v}} d \sigma \int_{\sigma}^{t} \eta_{n-1}(\tau) d \tau+M_{L} M_{f} \int_{0}^{t} \eta_{n-1}(\tau) d \tau .
$$

После перемены порядка интегрирования и вычисления квадратуры по $\sigma$ это переписывается в виде

$$
\eta_{n}(t) \leqslant M_{L} M_{f} \int_{0}^{t} e^{\tau M_{v}} \eta_{n-1}(\tau) d \tau,
$$


откуда следует более грубое, но и более простое неравенство:

$$
\eta_{n}(t) \leqslant M_{T} \int_{0}^{t} \eta_{n-1}(\tau) d \tau, \quad M_{T}=M_{f} M_{L} e^{T M_{v}}, \quad 0 \leqslant t \leqslant T .
$$

Отсюда, применяя индукцию, выводим оценку

$$
\eta_{n}(t)=\max _{x \in \bar{D}}\left|h_{n}(t) x-h_{n-1}(t) x\right| \leqslant \frac{\left(t M_{T}\right)^{n} \mu_{T}}{n !}, \quad \mu_{T}=\max _{(x, t) \in Q_{T}}\left|h_{0}(t) x-x\right| .
$$

Теперь ясно, что ряд $\sum_{n=0}^{\infty} \eta_{n}(t)$ сходится равномерно для $t \in[0, T]$, а вместе с ним и ряд (3.6) сходится на $Q_{T}$ равномерно.

Итак, последовательность $\left\{\theta_{n}\right\}$ равномерно на $Q_{T}$ сходится к некоторой функции $\theta$. Тогда и последовательность $\left\{\boldsymbol{v}_{n}\right\}, \boldsymbol{v}_{n}=L \theta_{n}$, сходится в пространстве $C\left([0, T], W_{p}^{(2)}(D)\right)$ к полю $\boldsymbol{v}=L \theta$ при любом $p>1$. По теореме вложения имеет место также и сходимость в $C\left([0, T], C^{1, \lambda}(D)\right)$ для любого $\lambda, 0 \leqslant \lambda<1$.

Из равномерной на компакте $Q_{T}$ сходимости последовательности полей $\left\{\boldsymbol{v}_{n}\right\}$ и последовательностей их производных $\left\{\partial \boldsymbol{v}_{n} / \partial x_{i}\right\}, i=1,2,3$, следует (см. [26]) сходимость соответствующих эволюционных операторов $g_{n}$, тоже равномерная на $Q_{T}$, вместе с первыми производными по $x, t$.

Таким образом, для пределов $\theta, \boldsymbol{v}$ имеем соотношения

$$
\theta(x, t)=f(h(t) x), \quad \boldsymbol{v}=L \theta,
$$

где $h(t)=g^{-1}(t), g(t)$ - эволюционный оператор поля $\boldsymbol{v}$. При этом $\theta \in C^{1}\left(Q_{T}\right)$ и удовлетворяет дифференциальному уравнению (1.1). Из известных результатов о существовании классического решения линеаризованной стационарной задачи для системы Стокса [7], [8] следует теперь, что и соотношения (1.2) выполнены в классическом смысле.

Итак, $(\theta, \boldsymbol{v})$ - классическое решение задачи (1.1)-(1.2), и теорема 3.1 доказана.

Если гладкость данных - границы $\partial D$ и начального поля температуры $f-$ улучшается, то улучшается и гладкость решения $(\theta, \boldsymbol{v})$. Можно было бы это установить, доказав сходимость последовательных приближений по более сильным нормам, скажем $W_{p}^{(\ell)}$ или $C^{k, \lambda}$. Проще, однако, непосредственно исследовать гладкость имеющегося решения. Так из свойства $\theta \in C^{1}\left(Q_{T}\right)$ при условии $\partial D \in C^{2}$ следует, что $\boldsymbol{v}=L \theta=G(\theta \boldsymbol{k})$ - непрерывно дифференцируемая по $t$ вектор-функция со значениями в $W_{p}^{(2)}$ или в $C^{1, \lambda}(D), 0<\lambda<1$ - любое число. Тогда отображение $(t, x) \mapsto h(t) x$ дважды непрерывно дифференцируемо по $x, t$. Выходит, что $\theta \in C^{2}\left(Q_{T}\right)$, если $f \in C^{2}(D)$. Аналогично можно продолжать и дальше. В итоге получаем, в частности, следующую теорему о $C^{\infty}$-гладкости.

Теорема 3.2. Пусть $D$ - ограниченная область в $\mathbb{R}^{3}$ с границей $\partial D$ класса $C^{\infty}$, и пусть $f \in C^{\infty}(D)$. Тогда решение $(\theta, \boldsymbol{v})$ задачи $(1.1),(1.2)$ бесконечно дифферениируемо по $x, t$. 


\section{§ 4. Обобщенные решения. Теорема единственности}

Среди многих возможных классов обобщенных решений ограничимся одним - наиболее широким классом, для которого еще можно доказать теорему единственности. Примечательно, что теоремы единственности решения задачи (1.1)-(1.5) для системы уравнений с частными производными и теорема единственности решения задачи Коши для обыкновенных дифференциальных уравнений движения жидких частиц справедливы при одних и тех же условиях. Ситуация аналогична той, которая возникает в проблеме единственности решения начально-краевой задачи для системы уравнений Эйлера [27], [28]. Как раз при $\theta$ из $L_{3}(D)$ (для каждого $t$ ) поле $\boldsymbol{v}(x, t)$ хотя и не удовлетворяет условию Липшица по $x$, подчинено условию типа Осгуда, которое тоже гарантирует единственность. Конечно, для $\theta$ из $L_{p}(D), p>3$, скорость $\boldsymbol{v}$ (при фиксированных $t$ ) непрерывно дифференцируема по $x$, так что выполняются классические условия единственности, но в случае $L_{p}(D), p<3$, для $\boldsymbol{v}$ получается лишь условие Гёльдера с показателем $\gamma, 0<\gamma<1$, а этого для единственности не хватает.

Определим обобщенное решение начально-краевой задачи (1.1)-(1.5) на отрезке времени $[0, T]$ как пару $(\theta, \boldsymbol{v})$, где $\theta(\cdot, t)$ - слабо непрерывная функция от $t \in[0, T]$ со значениями в $L_{3}(D)$, поле скоростей $\boldsymbol{v}=L \theta$, а уравнение (1.1) выполняется в следующем обобщенном смысле: для любой гладкой функции $\eta$ на $Q_{T}$ справедливо интегральное тождество

$$
\int_{0}^{T} \int_{D} \theta\left(\frac{\partial \eta}{\partial t}+v_{j} \frac{\partial \eta}{\partial x_{j}}\right) d x d t=\int_{D} \theta(x, T) \eta(x, T) d x-\int_{D} f(x) \eta(x, 0) d x
$$

Таким образом, уравнение (1.1) - или уравнение (3.1) - выполнено в смысле пространства обобщенных функций $W_{3}^{(-1)}(D)$. Последнее состоит из распределений $\varphi=\operatorname{div} g, g \in L_{3}(D)$, и норма в нем определяется равенством

$$
\|\varphi\|_{W_{3}^{(-1)}}=\sup \left\{\int_{D} g \cdot \nabla \eta d x: \eta \in C^{\infty}(D), \int_{D}|\nabla \eta|^{3 / 2} d x=1\right\} .
$$

ТЕОРема 4.1 (единственности). Существует не более одного обобщенного решения задачи (1.1)-(1.2).

ДОКАЗАТЕЛЬСТво. Если $\left(\theta^{\prime}, \boldsymbol{v}^{\prime}\right)$ и $\left(\theta^{\prime \prime}, \boldsymbol{v}^{\prime \prime}\right)$ - обобщенные решения, то для разностей $\varphi=\theta^{\prime}-\theta^{\prime \prime}, \boldsymbol{u}=\boldsymbol{v}^{\prime}-\boldsymbol{v}^{\prime \prime}$ имеем равенства $\boldsymbol{u}=L \varphi$ и

$$
\frac{\partial \varphi}{\partial t}=-\frac{\partial}{\partial x_{j}}\left(u_{j} \theta^{\prime}+v_{j}^{\prime \prime} \varphi\right)
$$

в смысле распределений. Определим функцию $\alpha$ как решение краевой задачи

$$
-\Delta \alpha=\varphi,\left.\quad \alpha\right|_{\partial D}=0,
$$


так что $\alpha=G_{1} \varphi$, где $G_{1}$ - оператор Грина. Согласно классическому результату А. И. Кошелева $G_{1}$ действует из $L_{p}$ в $W_{p}^{(2)}, p>1$, ограниченно, так что $\alpha \in$ $C\left([0, T], W_{3}^{(2)}(D)\right)$. Из (4.2) следует равенство

$$
\frac{\partial \alpha}{\partial t}=-G_{1} D_{j}\left(u_{j} \theta^{\prime}+v_{j}^{\prime \prime} \varphi\right), \quad D_{j}=\frac{\partial}{\partial x_{j}},
$$

из которого явствует, что вектор-функция $t \mapsto \alpha(\cdot, t)$ со значениями в энергетическом пространстве $H_{-\Delta}$ сильно непрерывно дифференцируема, так как операторы $G_{1} D_{j}, j=1,2,3$, действуют ограниченно из $L_{2}(D)$ в $H_{-\Delta}$. Умножая (4.4) скалярно на $\alpha$ в $H_{-\Delta}$ (или (4.2) - на $\alpha$ скалярно в $L_{2}(D)$ ), после интегрирования по частям приходим к соотношению

$$
\frac{1}{2} \frac{d}{d t} \int_{D}(\nabla \alpha)^{2} d x=\int_{D} u_{j} \theta^{\prime} \frac{\partial \alpha}{\partial x_{j}} d x+\int_{D} \frac{\partial v_{i}^{\prime \prime}}{\partial x_{j}} \frac{\partial \alpha}{\partial x_{i}} \frac{\partial \alpha}{\partial x_{j}} d x \equiv J_{1}+J_{2} .
$$

Для оценки первого слагаемого применим сначала неравенство Гёльдера с показателями $6,3,2$ и теорему вложения $H_{1}$ в $L_{6}$. Имеем

$$
\left|J_{1}\right| \leqslant M^{\prime} C_{6}\|\nabla \alpha(\cdot, t)\|_{L_{2}} \cdot\|\boldsymbol{u}(\cdot, t)\|_{H_{1}}, \quad M^{\prime}=\sup _{0 \leqslant t \leqslant T}\left\|\theta^{\prime}(\cdot, t)\right\|_{L_{3}},
$$

где $C_{6}$ - верхняя граница нормы оператора вложения $H_{1}$ в $L_{6}$. Далее, из представления $\boldsymbol{u}=L \varphi=-L \Delta \alpha=-L D_{j}\left(D_{j} \alpha\right)$ непосредственно получаем неравенство

$$
\|\boldsymbol{u}\|_{H_{1}} \leqslant\|\nabla \alpha\|_{L_{2}} .
$$

Пользуясь этим, из (4.6) выводим оценку

$$
\left|J_{1}\right| \leqslant M_{1}\|\nabla \alpha(\cdot, t)\|_{L_{2}}^{2}, \quad M_{1}=M^{\prime} C_{6} .
$$

Со вторым слагаемым $J_{2}$ дела обстоят несколько сложнее. Заметим, что $\boldsymbol{v}^{\prime \prime} \in W_{3}^{(2)}(D)$ при любом фиксированном $t$ в силу коэрцитивности оператора Стокса в $L_{3}(D)$ и равенства $\boldsymbol{v}^{\prime \prime}=L \theta^{\prime \prime}$. По теореме вложения Соболева [29], [30] $\boldsymbol{v}^{\prime \prime} \in W_{p}^{(1)}$ для любого $p>1$, а согласно усилению, полученному в [2], выполняется неравенство

$$
\left\|\nabla \boldsymbol{v}^{\prime \prime}\right\|_{L_{p}} \leqslant C p^{2 / 3}
$$

с постоянной $C$, не зависящей от $p, p \geqslant 1$, и от $t \in[0, T]$. Аналогичным образом из (4.3) получаем неравенство

$$
\|\nabla \alpha\|_{L_{p}} \leqslant C_{1} p^{2 / 3}
$$

где $C_{1}$ тоже не зависит от $p$ при $p \geqslant 1$ и от $t \in[0, T]$. Далее, впрочем, это неравенство нужно лишь при фиксированном $p=4$. В двумерном случае показатель в (4.8) и (4.9) вместо $2 / 3$ есть $1 / 2$. Константы $C$ и $C_{1}$ включают в себя множители $\left\|\theta^{\prime \prime}\right\|_{L_{3}}=\|f\|_{L_{3}}$ и $\|\varphi\|_{L_{3}} \leqslant 2\|f\|_{L_{3}}$.

Применяя для оценки интеграла $J_{2}$ неравенство Гёльдера, получаем

$$
\begin{aligned}
\left|J_{2}\right| & \leqslant \int_{D}\left|\nabla \boldsymbol{v}^{\prime \prime}\right| \cdot|\nabla \alpha|^{2} d x=\int_{D}\left|\nabla \boldsymbol{v}^{\prime \prime}\right| \cdot|\nabla \alpha|^{2-2 \varepsilon} \cdot|\nabla \alpha|^{2 \varepsilon} d x \\
& \leqslant\left\|\nabla \boldsymbol{v}^{\prime \prime}\right\|_{L_{2 / \varepsilon}(D)} \cdot z^{2-2 \varepsilon} \cdot\|\nabla \alpha\|_{L_{4}(D)}^{2 \varepsilon},
\end{aligned}
$$

где $z=z(t)=\|\nabla \alpha(\cdot, t)\|_{L_{2}(D)}$, а $\varepsilon, 0<\varepsilon<1,-$ произвольное число. 
Используя (4.8) и (4.9), отсюда выводим неравенство

$$
\left|J_{2}\right| \leqslant M_{2} \varepsilon^{-2 / 3} z^{2-2 \varepsilon},
$$

где $M_{2}$ не зависит от $\varepsilon$ и от $t \in[0, T]$, но, конечно, может зависеть от $T$.

Применяя (4.7) и (4.10), из (4.5) получаем дифференциальное неравенство

$$
z \dot{z} \leqslant M_{1} z^{2}+M_{2} \varepsilon^{-2 / 3} z^{2-2 \varepsilon} .
$$

Как известно (см., например, [26; гл. $3, \S 2]), z(t)$ не превосходит максимального решения $z_{*}$ задачи Коши

$$
z_{*} \dot{z}_{*}=M_{1} z_{*}^{2}+M_{2} \varepsilon^{-2 / 3} z_{*}^{2-2 \varepsilon}, \quad z_{*}(0)=0 .
$$

Всякое иное решение обращается в нуль при $t \in\left[0, t_{0}\right]$ для некоторого $t_{0}$, а при $t>t_{0}$ есть $z_{*}\left(t-t_{0}\right)$. Если $z_{*}-$ ненулевое решение, то $z_{*}(t)>0$ при $t>0$. Сокращая уравнение (4.12) на $z_{*}^{2-2 \varepsilon}$, получаем

$$
\frac{d}{d t} z_{*}^{2 \varepsilon}=2 \varepsilon M_{1} z_{*}^{2 \varepsilon}+2 \varepsilon^{1 / 3} M_{2}, \quad z_{*}(0)=0 .
$$

Решая эту линейную задачу Коши для $z_{*}^{2 \varepsilon}$, находим

$$
z_{*}^{2 \varepsilon}(t)=\left(2 M_{2} \varepsilon^{1 / 3} t\right) \frac{e^{2 \varepsilon M_{1} t}-1}{2 \varepsilon M_{1} t} .
$$

Пользуясь тем, что функция $\left(e^{s}-1\right) / s$ монотонно возрастает, оцениваем последнюю дробь, полагая $\varepsilon=1$ и $t=T$. В результате получаем

$$
z(t) \leqslant z_{*}(t) \leqslant\left(2 M M_{2} \varepsilon^{1 / 3} t\right)^{1 /(2 \varepsilon)}, \quad M=\frac{e^{M_{1} T}-1}{2 M_{1} t} .
$$

Полагая здесь $\varepsilon \rightarrow 0$, выводим, что $z(t)=0$ для $t \in[0, T]$, а ввиду произвольности $T$ и для всех $t \geqslant 0$. Аналогично получается, что $z(t) \equiv 0$ и для $t \leqslant 0$. Теорема 4.1, таким образом, доказана.

Другой способ обработки неравенства (4.11) состоит в том, чтобы перейти в правой части к инфимуму по $\varepsilon, 0<\varepsilon<1$. Тогда получается дифференциальное неравенство

$$
z \dot{z} \leqslant M_{1} z^{2}+M_{2}(3 e)^{2 / 3} z^{2}\left(\ln z^{-1}\right)^{2 / 3}
$$

при условии, что $z(t) \leqslant 1$. Отсюда, применяя теорему о дифференциальном неравенстве и пользуясь тем, что правая часть в (4.13) удовлетворяет условию единственности Осгуда, снова получаем, что $z(t) \equiv 0$.

Было бы очень интересно (это по-настоящему трудная задача) расширить класс единственности, указанный в теореме 4.1, поскольку существование получается при гораздо более общих условиях.

Рассмотрим, например, задачу (1.1)-(1.2) при $f \in L_{p}(D), p \geqslant 2$ (можно было бы и для $\left.f \in L_{p}(D), p>1\right)$. Тогда обобщенное решение, скажем для $t>0$, 
можно построить как предел при исчезающей теплопроводности $\delta$ решений начально-краевой задачи

$$
\begin{gathered}
\frac{d \theta_{\delta}}{d t}=\delta \Delta \theta_{\delta},\left.\quad \theta_{\delta}\right|_{\partial D}=0,\left.\quad \theta_{\delta}\right|_{t=0}=f, \\
\boldsymbol{v}_{\delta}=L \theta_{\delta}, \quad \frac{d}{d t}=\frac{\partial}{\partial t}+\boldsymbol{v}_{\delta} \cdot \nabla .
\end{gathered}
$$

При $\delta>0$ однозначная глобальная разрешимость в классе $C\left([0, T], L_{p}(D)\right) \cap$ $\left.L_{2}\left([0, T], H_{-\Delta}\right)\right)$ доказывается для этой задачи стандартно (см. [9], [31]), и непосредственно выводится равенство

$$
\frac{1}{p} \frac{d}{d t} \int_{D}\left|\theta_{\delta}\right|^{p} d x=-\delta(p-1) \int_{D}\left(\nabla \theta_{\delta}\right)^{2}\left|\theta_{\delta}\right|^{p-2} d x
$$

из которого следуют априорные оценки

$$
\begin{gathered}
\left\|\theta_{\delta}(\cdot, t)\right\|_{L_{p}(D)} \leqslant\|f\|_{L_{p}(D)}, \\
\delta(p-1) \int_{0}^{t} \int_{D}\left(\nabla \theta_{\delta}\right)^{2} \theta_{\delta}^{p-1} d x d s \leqslant \frac{1}{p}\|f\|_{L_{p}(D)}^{p} .
\end{gathered}
$$

Еще одно существенное соотношение получается из (4.14) умножением на произвольную гладкую функцию $\Phi(x), x \in D, \Phi=0$ на $\partial D$, и интегрированием по $D$ :

$$
\frac{d}{d t} \int_{D} \theta_{\delta}(c, t) \Phi(x) d x=\int_{D} \theta_{\delta} \boldsymbol{v}_{\delta} \cdot \nabla \Phi d x+\delta \int_{D} \nabla \theta_{\delta} \cdot \nabla \Phi d x .
$$

Для обобщенного решения задачи (4.14) выполняется интегральное тождество

$$
\int_{D}\left(\frac{\partial \eta}{\partial t}+\boldsymbol{v}_{\delta} \cdot \nabla \eta\right) d x d t=\delta \int_{0}^{T} \int_{D} \nabla \theta_{\delta} \eta d x d t-\int_{D} f(x) \eta(x, 0) d x
$$

при любом $t>0$ и для любой гладкой функции $\eta$ на $Q_{T}$ такой, что $\eta=0$ на $\partial D$ и при $t=T$.

Из (4.15) и (4.16), как обычно, получается компактность семейства $\left\{\theta_{\delta}\right\}$ в $L_{2}\left(Q_{T}\right)$. А тогда и семейство $\left\{\boldsymbol{v}_{\delta}\right\}, \boldsymbol{v}_{\delta}=L \theta_{\delta}$, компактно. Переходя в (4.17) к пределу по надлежащей подпоследовательности $\delta_{k} \rightarrow 0$, для пределов $\theta, \boldsymbol{v}$ получаем (4.1), правда, пока лишь при дополнительном условии $\eta=0$ на $\partial D$ и для $t=T$. После этого (4.1) в полном объеме доказывается известными приемами: 1$)$ переходим от $\eta(x, t)$ к $\tau(t) \eta(x, t), \tau(T)=0$, и т.д., чтобы избавиться от условия $\eta(x, T)=0 ; 2)$ если $\eta$ - произвольная гладкая на $Q_{T}$ функция, записываем (4.1) для пробной функции $\chi(x) \cdot \eta(x, t)$, где $\chi$ - гладкая и финитная в $D$ функция. В результате приходим к равенству вида

$$
\int_{D} \chi F d x+\int_{D} \nabla \chi \cdot g d x=0
$$

где использованы обозначения

$$
\begin{gathered}
F(x)=\int_{0}^{T} \theta\left(\frac{\partial \eta}{\partial t}+\boldsymbol{v} \cdot \nabla \eta\right) d t-\theta(x, T) \eta(x, T)+f(x) \eta(x, 0), \\
g_{j}(x)=\int_{0}^{T} v_{j} \eta \theta d t, \quad j=1,2,3 .
\end{gathered}
$$


Это означает, что $\operatorname{div} g=F$ в смысле распределений. Отвлечемся временно от конкретного вида $g$ и $F$.

ЛЕмма 4.1. Пусть для любой функиии $\chi \in C_{0}^{\infty}$ выполнено (4.18), где $g$ векторное поле, $F$ - функиия, заданные на ограниченной области $D \subset \mathbb{R}^{3}$. Пусть $g, F$ - класса $L_{1}$ и поле $g$ исчезает на граниче $\partial D$ в следующем обобщенном смысле:

$$
\int_{D_{\varepsilon}}|g(x)| d x=o(\varepsilon), \quad \varepsilon \rightarrow 0,
$$

где $D_{\varepsilon}-$ пограничный слой толщины $\varepsilon$, m.e. $D_{\varepsilon}=\{x \in D \mid \rho(x, \partial D)<\varepsilon\}$.

Тогда выполняется равенство

$$
\int_{D} F d x=0
$$

ДокАЗАТЕльство. Для каждого достаточно малого $\varepsilon>0$ определим срезающую функцию $\chi_{\varepsilon}$ класса $C^{\infty}$ с носителем в $D_{\varepsilon}$ такую, что всюду в $D$ выполняются неравенства $\left|\chi_{\varepsilon}(x)\right| \leqslant 1,\left|\nabla \chi_{\varepsilon}(x)\right| \leqslant C / \varepsilon(C$ не зависит от $\varepsilon)$, а в остальном $\chi_{\varepsilon}$ произвольна. Существование таких функций хорошо известно.

Положим в (4.18) $\chi=\chi_{\varepsilon}$ и покажем, что при $\varepsilon \rightarrow 0$ второе слагаемое стремится к нулю. Действительно,

$$
\left|\int_{D} \nabla \chi_{\varepsilon} \cdot g d x\right| \leqslant \frac{C}{\varepsilon} \int_{D_{\varepsilon}}|g(x)| d x \rightarrow 0
$$

при $\varepsilon \rightarrow 0$ в силу (4.19).

Далее, при $\varepsilon \rightarrow 0$

$$
\int_{D} \chi_{\varepsilon} F d x \rightarrow \int_{D} F d x
$$

по теореме Лебега об ограниченной сходимости, так как $\left|\chi_{\varepsilon}(x)\right| \leqslant 1$ и $\chi_{\varepsilon}(x) \rightarrow 1$ почти всюду в $D$.

Итак, (4.20) выполняется и лемма 4.1 доказана.

Проверим теперь, что определенное выше поле $g$ удовлетворяет условию (4.19). Имеем

$$
\int_{D_{\varepsilon}}|g(x)| d x \leqslant \int_{0}^{T} \int_{D_{\varepsilon}}|\boldsymbol{v}||\eta||\theta| d x d t .
$$

Этот интеграл не превосходит величины

$$
\|\eta\|_{C} \cdot\|\theta\|_{L_{2}\left(Q_{T, \varepsilon}\right)} \cdot\|\boldsymbol{v}\|_{L_{2}\left(Q_{T, \varepsilon}\right)},
$$

где $Q_{T, \varepsilon}=D_{\varepsilon} \times[0, T]$.

Далее воспользуемся тем, что $\boldsymbol{v}=L \theta \in H_{1}$, и применим неравенство Лерэ [32]

$$
\int_{D_{\varepsilon}} \boldsymbol{v}^{2} d x \leqslant C^{2} \varepsilon^{2} \int_{D_{\varepsilon}}(\nabla \boldsymbol{v})^{2} d x,
$$

$C>0$ и зависит лишь от области $D$. Из (4.21)-(4.23) получаем оценку

$$
\int_{D_{\varepsilon}}|g(x)| d x \leqslant C \varepsilon\|\eta\|_{C} \cdot\|\theta\|_{L_{2}\left(Q_{T, \varepsilon}\right)} \cdot\|\boldsymbol{v}\|_{L_{2}\left(Q_{T, \varepsilon}\right)},
$$

из которой и вытекает (4.19). 
Из леммы 4.1 теперь следует, что выполняется (4.20) для $F$ из (4.18), а это значит, что (4.1) выполнено для всех гладких $\eta$.

В итоге приходим к следующей теореме существования.

Tеорема 4.2. Пусть $f \in L_{p}(D), p \geqslant 2$. Тогда существует обобщенное решение задачи (1.1)-(1.2) - т.е. пара $(\theta, \boldsymbol{v})$, где $\theta$ - слабо непрерывная функиия от $t \in \mathbb{R}$ со значениями в $L_{p}(D), \boldsymbol{v}=L \theta$, такая, что (4.1) выполняется для любого $T$ и произвольной гладкой функиии $\eta$ на $Q_{T}$.

Примерно та же техника позволяет рассмотреть и обобщенные решения в $L_{p}$ при любом $p>1$, но единственность получается лишь при $p \geqslant 3(p \geqslant 2$ в двумерном случае).

Отметим в заключение этого параграфа, что для задачи (1.1)-(1.2) оказывается возможным определить обобщенное решение в классе обобщенных функций. Дело в том, что если положить в (4.1) $\theta=\operatorname{div} \alpha, \alpha-$ касательное к $\partial D$ поле, то все производные с $\alpha$ можно перебросить интегрированием по частям, например

$$
\int_{D} \theta v_{j} \frac{\partial \eta}{\partial x_{j}} d x=-\int_{D} \alpha_{i}\left(\frac{\partial v_{j}}{\partial x_{i}} \frac{\partial \eta}{\partial x_{j}}+v_{j} \frac{\partial^{2} \eta}{\partial x_{i} \partial x_{j}}\right) d x
$$

Получающееся интегральное тождество можно принять за определение обобщенного решения, предполагая при этом, что $\alpha$ - слабо непрерывная векторфункция от $t$ со значениями в $L_{1}(D)$ (можно и в $L_{p}$ ). А тогда $\boldsymbol{v}=L \theta=$ $L D_{i} \alpha_{i} \in H_{1}$ для любого $t$, так что определение оказывается корректным.

Несколько иной вариант: сохраняя соотношение $\boldsymbol{v}=L \theta$, предполагать лишь, что $\boldsymbol{v} \in H_{1}$ для каждого $t$.

\section{$\S 5$. Поведение решений при $t \rightarrow \infty$. Затухание движений}

В этом параграфе доказано, что для всякого решения $(\theta, \boldsymbol{v})$ задачи $(1.1)-(1.2)$ при $t \rightarrow+\infty$ или $t \rightarrow-\infty$ скорость $\boldsymbol{v}$ затухает по норме $H_{1}$, а температура $\theta$ стремится к некоторой точке на подпространстве равновесий $\mathscr{E}$ слабо в $L_{2}(D)$.

Для краткости будем дальше вести речь о гладких решениях; результаты, конечно, сохраняются и для обобщенных решений, лишь бы для них были определены фигурирующие в оценках величины. Ввиду обратимости (предложение 2.1) имеем право ограничиться предельным переходом при $t \rightarrow+\infty$.

Будем далее пользоваться следующими определениями и обозначениями: $H=L_{2}(D), \mathscr{E}$ - подпространство в $H$, состоящее из равновесных профилей температуры $\theta$. Последние определяются, вместе с соответствующими полями давления $P$, как обобщенные решения уравнения $\nabla P=\theta \boldsymbol{k}$ (см. (2.5)). Согласно предложению 2.5 , когда все сечения области $D$ горизонтальными плоскостями связны, это попросту функции $\theta=\theta(z)$ из $H$, не зависящие от $x_{1}, x_{2}$. В общем случае функция $\theta \in \mathscr{E}$ определяется из условия

$$
\int_{D} \theta \Phi_{3} d x=0
$$


для любого поля $\Phi \in H_{1}$. Через $H_{1}$ обозначено энергетическое пространство оператора Стокса. Равенство (5.1) выполняется и для всех $\Phi \in S_{2}$. Гильбертово пространство $S_{2}$ определяется как замыкание множества финитных в $D$ соленоидальных векторных полей в $L_{2}$-метрике (см. [9; гл. $\left.1, \S 5\right]$, [33], [34]). Пространство $H_{1}$ естественно вложено в $S_{2}$ как всюду плотное линейное многообразие. При этом оператор вложения вполне непрерывен.

Через $P_{0}, P^{0}$ обозначим ортопроекторы в $H$ на $\mathscr{E}$ и соответственно на его ортогональное дополнение $H^{0}=H \ominus \mathscr{E}$. Выполняется равенство $P_{0}+P^{0}=I$. Нетрудно указать явное представление проектора $P_{0}$. Например, в случае, когда все сечения $S_{z}$ связны, $\bar{\theta}(z)=\left(P_{0} \theta\right)(z)$ получается осреднением на $S_{z}$ :

$$
\bar{\theta}(z)=\frac{1}{\operatorname{mes} S_{z}} \int_{S_{z}} \theta\left(x_{1}, x_{2}, z\right) d x_{1} d x_{2} .
$$

Через $H_{-}$обозначим негативное, порядка -1 , гильбертово пространство обобщенных функций, получаемое замыканием $H^{0}$ в метрике скалярного произведения:

$$
\left(\theta^{\prime}, \theta^{\prime \prime}\right)_{-}=\left(L \theta^{\prime}, L \theta^{\prime \prime}\right)_{1}=\left(\theta^{\prime}, \boldsymbol{k} \cdot G\left(\theta^{\prime \prime} \boldsymbol{k}\right)\right), \quad \theta^{\prime}, \theta^{\prime \prime} \in H^{0} .
$$

Последнее скалярное произведение - в $H$, а предыдущее - в $H_{1}$. Здесь $G-$ оператор Грина системы Стокса, $L \theta=G(\theta \boldsymbol{k})$.

ЛЕмма 5.1. Для любого решения $(\theta, \boldsymbol{v})$ задачи (1.1)-(1.2) справедливо равенство

$$
\left\|\theta^{0}(\cdot, t)\right\|_{-}=\|\boldsymbol{v}(\cdot, t)\|_{1}, \quad \theta^{0}=P^{0} \theta, \quad t \in \mathbb{R} .
$$

ДокАзАтельство. Согласно (1.2) для любого $\Phi \in H_{1}$ выполняется равенство

$$
(\boldsymbol{v}(t), \Phi)_{1}=\left(\theta(t), \Phi_{3}\right)=\left(\theta^{0}(t), \Phi_{3}\right) .
$$

Здесь и далее пишем $\boldsymbol{v}(t), \theta(t)$ и т.д. вместо $\boldsymbol{v}(x, t), \boldsymbol{v}(\cdot, t)$ и т.п. Взяв в $(5.3)$ верхнюю грань по $\Phi$ на единичном шаре в $H_{1}$, приходим к $(5.2)$, что и требовалось.

Определим оператор $Q: H_{1} \rightarrow H$, полагая $Q \Phi=\Phi_{3}$. Нетрудно убедиться в том, что его гильбертов сопряженный оператор $Q^{*}: H \rightarrow H_{1}$ действует по правилу $Q^{*} \theta=G(\theta k)$ для $\theta \in H$.

Лемма 5.2. Подпространство $\mathscr{E}$ есть ядро оператора $Q^{*}$, а подпространство $H^{0}$ - замыкание образа оператора $Q$ :

$$
\mathscr{E}=\operatorname{ker} Q^{*}, \quad H^{0}=\overline{\operatorname{im} Q} .
$$

ДоКАЗАТЕЛЬСтво с очевидностью следует из определения (5.1) подпространства $\mathscr{E}$.

Лемма 5.3. Подпространство $\mathscr{E}$ - притягивающее в том смысле, что для любого решения $(\theta, \boldsymbol{v})$ задачи (1.1)-(1.2) при $t \rightarrow+\infty$

$$
\|\boldsymbol{v}(t)\|_{1} \rightarrow 0, \quad \theta^{0}(t)=P^{0} \theta(t) \rightarrow 0
$$

слабо в $H$ и по норме $H_{-}$. 
ДокАЗАТЕЛЬСтво. Умножая (1.2) скалярно в $L_{2}$ на $\boldsymbol{v}$, получаем равенство

$$
\|\boldsymbol{v}(t)\|_{1}^{2}=\left(\theta(t), \boldsymbol{v}_{3}(t)\right)=\left(\theta^{0}(t), \boldsymbol{v}_{3}(t)\right)
$$

Применение неравенства Коши-Буняковского и неравенства Фридрихса

$$
\|\boldsymbol{v}(t)\|_{1} \geqslant \gamma\|\boldsymbol{v}(t)\|
$$

с постоянной $\gamma$, зависящей лишь от области $D$ (см. [35]), дает оценку

$$
\|\boldsymbol{v}(t)\|_{1} \leqslant \gamma^{-1}\|\theta(t)\|=\gamma^{-1}\|f\| .
$$

Последнее равенство следует из (2.11). Таким образом, вектор-функция $\boldsymbol{v}(t)$ ограничена в $H_{1}$ для $t \in \mathbb{R}_{+}$.

Теперь докажем, что $\|\boldsymbol{v}(t)\|_{H_{1}} \rightarrow 0$ при $t \rightarrow+\infty$. Как известно из анализа (и вытекает из приведенной ниже для полноты картины леммы 5.4), ограниченность производной $\frac{d}{d t}\|\boldsymbol{v}(t)\|_{H_{1}}$ вместе с квадратичной суммируемостью функции $\|\boldsymbol{v}(t)\|_{H_{1}}$ на $\mathbb{R}_{+}$, установленной в предложении 2.7 (см. (2.10)), дают затухание этой функции при $t \rightarrow+\infty$.

Докажем, что справедливо равенство

$$
\frac{d}{d t}\|\boldsymbol{v}(t)\|_{H_{1}}^{2}=2 \int_{D}(\boldsymbol{v}, \nabla) \boldsymbol{v} \cdot \theta \boldsymbol{k} d x .
$$

Действительно, дифференцируя равенство (5.5), находим

$$
\frac{d}{d t}\|\boldsymbol{v}(t)\|_{H_{1}}^{2}=\int_{D}\left[\boldsymbol{v}_{t}+(\boldsymbol{v}, \nabla) \boldsymbol{v}\right] \cdot \theta \boldsymbol{k} d x .
$$

Поскольку $\boldsymbol{v}=G(\theta \boldsymbol{k})$, с учетом (1.1) получаем равенство $\boldsymbol{v}_{t}=-G(\boldsymbol{v} \cdot \nabla \theta \boldsymbol{k})$. Оператор $G$ самосопряжен. Поэтому

$$
\int_{D} \boldsymbol{v}_{t} \cdot \theta \boldsymbol{k} d x=-\int_{D} G(\theta \boldsymbol{k}) \cdot(\boldsymbol{v} \cdot \nabla \theta \boldsymbol{k}) d x=-\int_{D} \boldsymbol{v} \cdot \frac{\partial}{\partial x_{i}}\left(v_{i} \theta \boldsymbol{k}\right) d x .
$$

Интегрируя по частям, получаем

$$
\int_{D} \boldsymbol{v}_{t} \cdot \theta \boldsymbol{k} d x=\int_{D}(\boldsymbol{v}, \nabla) \boldsymbol{v} \cdot \theta \boldsymbol{k} d x
$$

что вместе с (5.8) приводит к равенству (5.7).

Учитывая, что $|(\boldsymbol{v}, \nabla) \boldsymbol{v}| \leqslant|\boldsymbol{v}||\nabla \boldsymbol{v}|$, и применяя для оценки правой части (5.7) неравенство Гёльдера с показателями $6,2,3$, получаем неравенство

$$
\left|\frac{d}{d t}\|\boldsymbol{v}(t)\|_{H_{1}}^{2}\right| \leqslant 2\|\boldsymbol{v}\|_{L_{6}}\|\boldsymbol{v}\|_{H_{1}}\|\theta\|_{L_{3}} .
$$

Воспользуемся неравенством вложения

$$
\|\boldsymbol{v}\|_{L_{6}} \leqslant C\|\boldsymbol{v}\|_{H_{1}}
$$


с абсолютной константой $C$. Из (5.9) и (5.10) выводим, что

$$
\left|\frac{d}{d t}\|\boldsymbol{v}(t)\|_{H_{1}}^{2}\right| \leqslant 2 C\|\boldsymbol{v}\|_{H_{1}}^{2}\|\theta\|_{L_{3}} .
$$

С учетом (5.6) и (2.11) окончательно получаем

$$
\left|\frac{d}{d t}\|\boldsymbol{v}(t)\|_{H_{1}}^{2}\right| \leqslant 2 C \gamma^{-2}\|f\|^{2}\|f\|_{L_{3}} .
$$

Из (5.12) и (5.6) вытекает также неравенство

$$
\left|\frac{d}{d t}\|\boldsymbol{v}(t)\|_{H_{1}}\right| \leqslant C \gamma^{-1}\|f\| \cdot\|f\|_{L_{3}} .
$$

Согласно лемме 5.4 из (2.10) и этого неравенства вытекает первое предельное равенство (5.4). Из него следует, что для любого $\Phi \in H_{1}$ существует предел

$$
\left(\theta(t), \Phi_{3}\right)=(\boldsymbol{v}(t), \Phi)_{H_{1}} \rightarrow 0
$$

при $t \rightarrow+\infty$. Согласно лемме 5.2 равенство (5.13) выполняется для всех $\Phi_{3} \in H^{0}$. Если к этому добавить ограниченность вектор-функции $\theta(t)$ в $H$, то можно утверждать, что $\theta^{0}(t)=P^{0} \theta(t) \rightarrow 0$ слабо в $H^{0}$. Лемма 5.3 , таким образом, доказана.

Заметим, что предельные равенства (5.4) можно доказать, не используя ограниченности $\|\boldsymbol{v}(t)\|_{H_{1}}$, неравенства (5.6). Действительно, согласно (2.10) и (5.11) сходятся интегралы

$$
\int_{0}^{\infty}\|\boldsymbol{v}(t)\|_{H_{1}}^{2} d t<\infty, \quad \int_{0}^{\infty}\left|\frac{d}{d t}\|\boldsymbol{v}(t)\|_{H_{1}}^{2}\right| d t<\infty .
$$

Из равенства

$$
\|\boldsymbol{v}(t)\|_{H_{1}}^{2}-\|\boldsymbol{v}(s)\|_{H_{1}}^{2}=\int_{s}^{t} \frac{d}{d \tau}\|\boldsymbol{v}(\tau)\|_{H_{1}}^{2} d \tau
$$

следует, что существует предел

$$
\lim _{t \rightarrow+\infty}\|\boldsymbol{v}(t)\|_{H_{1}}^{2}=\|\boldsymbol{v}(s)\|_{H_{1}}^{2}+\int_{s}^{\infty} \frac{d}{d \tau}\|\boldsymbol{v}(\tau)\|_{H_{1}}^{2} d \tau .
$$

Но так как $\|\boldsymbol{v}(t)\|_{H_{1}}^{2}$ - интегрируемая функция, этот предел может быть равен только нулю.

ЛЕмма 5.4 [36]. Пусть и(t) - вещественная функиия на луче $\mathbb{R}_{+}=[0,+\infty)$, обладающая ограниченной производной $\dot{u}(t)$ и принадлежашая $L_{2}(0, \infty)$. Тогда для любого $\tau \geqslant 0$ справедливо неравенство

$$
\sup _{t \geqslant \tau}|u(t)|^{3} \leqslant \frac{27}{4} \int_{\tau}^{\infty} u^{2}(s) d s \sup _{t \geqslant \tau}|\dot{u}(t)| .
$$


ДокАЗАтЕЛьСтво. Пусть $\sup _{t \geqslant \tau}|\dot{u}(t)|=c>0$. Если $s_{0} \geqslant \tau$ и $\left|u\left(s_{0}\right)\right|=$ $\eta>0$, то из неравенств $|u(s)| \geqslant\left|u\left(s_{0}\right)\right|-\left|u(s)-u\left(s_{0}\right)\right|$ и $\left|u(s)-u\left(s_{0}\right)\right| \leqslant c\left|s-s_{0}\right|$ при любом $s \geqslant \tau$ следует, что $|u(s)| \geqslant 2 \eta / 3$, если $\left|s-s_{0}\right| \leqslant \eta /(3 c)$.

Далее имеем

$$
\int_{\tau}^{\infty} u^{2}(s) d s \geqslant \int_{s_{0}}^{s_{0}+\eta /(3 c)} u^{2}(s) d s \geqslant\left(\frac{2 \eta}{3}\right)^{2} \cdot \frac{\eta}{3 c}=\frac{4 \eta^{3}}{27 c} .
$$

Взяв здесь супремум по $s_{0} \geqslant \tau$, придем к неравенству (5.14). Утверждение доказано.

Лемма 5.5. Для любого решения $(\theta, \boldsymbol{v})$ задачи (1.1), (1.2) существует функция $\theta_{+} \in \mathscr{E}$ такая, что $\theta(t) \rightarrow \theta_{+}$при $t \rightarrow+\infty$ слабо в $H$.

ДокАЗАТЕЛЬСтво. Для любой функции $h \in \mathscr{E} \cap C^{1}$, применяя (1.1), получаем соотношение

$$
\frac{d}{d t}(\theta(t), h)=\left(\theta(t), \frac{\partial h}{\partial z} \boldsymbol{v}_{3}(t)\right)
$$

Поэтому существует

$$
\lim _{t \rightarrow+\infty}(\theta(t), h)=(f, h)+\int_{0}^{\infty}\left(\theta(t), \frac{\partial h}{\partial z} \boldsymbol{v}_{3}(t)\right) d t
$$

если этот интеграл сходится. Докажем, что так оно и есть. Для этого вспомним (см. [9; гл. $1, \S 5]$ ), что всякое поле $\boldsymbol{v} \in H_{1}$ можно представить в виде $\boldsymbol{v}=\operatorname{rot} B$, причем векторный потенциал $B$ исчезает на границе $\partial D$ и допускает оценку

$$
\|B\|_{W_{2}^{(1)}(D)} \leqslant C\|\boldsymbol{v}\|_{1},
$$

где $C$ зависит только от области $D$ (на самом деле, такая же оценка имеется для вектора $B$ и в $\left.W_{2}^{(2)}\right)$.

Если $h \in C^{3} \cap \mathscr{E}$, то поле $\Phi=\operatorname{rot}\left(\frac{\partial h}{\partial z} B\right) \in H_{1}$ и $\Phi_{3}=\frac{\partial h}{\partial z} \boldsymbol{v}_{3}$. Кроме того, выполнено неравенство

$$
\|\Phi\|_{1} \leqslant C_{1}\|\boldsymbol{v}\|_{1}
$$

с константой $C_{1}$, зависящей лишь от $D$.

Теперь, используя определение пространства $H_{-}$и свойства построенного поля $\Phi$, получаем неравенство

$$
\left|\left(\theta(t), \frac{\partial h}{\partial z} \boldsymbol{v}_{3}(t)\right)\right|=\left|\left(\theta(t), \Phi_{3}(t)\right)\right| \leqslant C_{1}\left\|\theta^{0}(t)\right\|_{-} \cdot\|\boldsymbol{v}(t)\|_{1}=C_{1}\|\boldsymbol{v}(t)\|_{1}^{2} .
$$

Сходимость интеграла в (5.15) следует поэтому из (2.10).

Таким образом, предел (5.15) существует для плотного в $\mathscr{E}$ множества функций $h \in H$. Так как вектор-функция $\theta(t)$ ограниченна в $H$, получается, что проекция $\theta_{0}(t)=P_{0} \theta(t)$ слабо сходится при $t \rightarrow+\infty$ к некоторой функции $\theta_{+} \in \mathscr{E}$. Но $\theta^{0}(t) \rightarrow 0$ слабо в $H$. Лемма 5.5 доказана.

Из лемм 5.1, 5.3 и 5.5 следует 
ТЕОРема 5.1. Для любого решения $(\theta, \boldsymbol{v})$ задачи (1.1), (1.2) движение жидкости затухает: $\|\boldsymbol{v}(t)\|_{1} \rightarrow 0$ при $t \rightarrow \mp \infty$, и существуют такие $\theta_{\mp} \in \mathscr{E}$, ито mемпература $\theta(t) \rightarrow \theta_{\mp}$ при $t \rightarrow \mp \infty$ слабо в $H$ и сильно в $H_{-}$.

Поскольку согласно предложению $2.2\|\theta(t)\|_{L_{p}}$ не зависит от времени, так что $\|\theta(t)\|_{L_{p}}=\|f\|_{L_{p}}$, можно утверждать, что в случае, когда $f \in L_{p}, p>2$, слабая сходимость $\theta(t)$ к $\theta_{\mp}$ при $t \rightarrow \mp \infty$ имеет место и в $L_{p}$. Если $f \in L_{\infty}$, то $\theta(t)$ слабо сходится во всех $L_{p}$. Дальнейшее увеличение требований регулярности к начальному полю температуры $f$ не приводит к усилению этого результата. Производные функции $\theta$, по-видимому, не затухают, а даже могут расти. Что касается скорости, то из представления $\boldsymbol{v}=L \theta$ следует, что при $t \rightarrow \infty$ она затухает в смысле слабой $W_{2}^{(2)}$-сходимости. Отсюда, в частности, вытекает, что $\boldsymbol{v}(x, t) \rightarrow 0$ при $t \rightarrow+\infty$ равномерно в $D$. Кроме того, можно оценить $\left\|\boldsymbol{v}_{t}(\cdot, t)\right\|_{1}$ следующим образом. Из равенства

$$
\left\|\boldsymbol{v}_{t}(t)\right\|_{1}^{2}=\int_{D} \theta v_{j} \frac{\partial v_{3 t}}{\partial x_{j}} d x
$$

применяя неравенство Гёльдера с показателями 3, 6, 2 и теорему вложения, получаем оценку

$$
\left\|\boldsymbol{v}_{t}(t)\right\|_{1} \leqslant\|f\|_{L_{3}} \cdot\|\boldsymbol{v}(t)\|_{L_{6}} \leqslant C\|f\|_{L_{3}} \cdot\|\boldsymbol{v}(t)\|_{1}
$$

из которой следует затухание нормы в левой части.

В заключение не откажем себе в удовольствии рассмотреть изученный здесь предельный случай для системы Лоренца

$$
\begin{cases}\dot{x}=-\sigma x+\sigma y, & \sigma=\mu / \delta, \\ \dot{y}=-x z++r x-y, & r=1 /(\mu \delta), \\ \dot{z}=-x y-b z . & \end{cases}
$$

Замена переменных $x=r x_{1}, y=r y_{1}, z=r\left(z_{1}+1\right), \tau=r t$ приводит ее к виду

$$
\begin{aligned}
\frac{1}{\mu^{2}} \frac{d x_{1}}{d \tau} & =-x_{1}+y_{1}, \\
\frac{d y_{1}}{d \tau} & =-x_{1} z_{1}-\frac{1}{r} y_{1}, \\
\frac{d z_{1}}{d \tau} & =x_{1} y_{1}-\frac{b}{r}\left(z_{1}+1\right) .
\end{aligned}
$$

В предельном случае $\mu=\infty, r=\infty$ имеем равенство $y_{1}=x_{1}$ и уравнения

$$
\begin{aligned}
& \frac{d y_{1}}{d \tau}=-y_{1} z_{1}, \\
& \frac{d z_{1}}{d \tau}=y_{1}^{2} .
\end{aligned}
$$

Решая эту систему в полярных координатах $y_{1}=\rho \cos \varphi, z_{1}=\rho \sin \varphi$, получаем, что $\rho=$ const, $\dot{\varphi}=-\rho \sin \varphi$ и в итоге

$$
\varphi(\tau)=2 \operatorname{arctg}\left(C e^{-\rho t}\right), \quad C=\text { const. }
$$


Примечательно, что выход на многообразие равновесий $\mathscr{E}$ (здесь это ось $z_{1}$ ) происходит экспоненциально и тем быстрее, чем больше начальная энергия системы. Аналогичный результат можно установить также для галёркинских аппроксимаций. Что касается полной системы, вряд ли можно дать квалифицированную оценку скорости сходимости поля температуры к предельному распределению. Во всяком случае для линейной задачи согласно результатам [22] затухание возмущения устойчивого равновесия может быть сколь угодно медленным.

\section{§ 6. Заключение. Дополнительные замечания}

В этом параграфе приводится ряд замечаний относительно дальнейшего развития настоящей работы и возможных обобщений.

Конвективные перевороты. Основной результат статьи - доказательство того факта, что при нулевой теплопроводности все движения жидкости затухают, при $t \rightarrow \mp \infty$ устанавливается равновесие с профилем температуры, зависящим лишь от вертикальной координаты $z$. При $t>0$ потенциальная энергия жидкости в поле тяжести уменьшается. Это означает, что холодные и тяжелые жидкие частицы в основном тонут, а горячие и легкие - всплывают. Естественно назвать такое движение конвективным переворотом.

Этот результат должен составить основу асимптотической теории конвекции жидкости при малой теплопроводности $\delta$ и большой вязкости $\mu$. При этом наиболее интересен случай, когда число Рэлея $r=1 /(\mu \delta)$ велико; тогда основное равновесие $\boldsymbol{v}_{0}=0, \theta_{0}=-z$ заведомо неустойчиво. Фактически речь здесь идет о турбулентной конвекции в этой асимптотической ситуации.

Конвективный переворот - быстрый процесс, поэтому малая теплопроводность $\delta$ не успевает существенно повлиять и с большой точностью поле температуры становится функцией лишь вертикальной переменной $z$, а скорость затухает. Для этого предельного режима, однако, не выполнено краевое условие $\left.\theta\right|_{\partial D}=-z$, так что, как бы ни было мало $\delta$, движение продолжается.

Дальнейший ход процесса поясним на примере жидкости, заполняющей горизонтальный слой $(-h / 2 \leqslant z \leqslant h / 2, h$ - толщина слоя), при условии периодичности полей $\boldsymbol{v}, P, \theta$ по $x_{1}$ и $x_{2}$ с заданными периодами $\ell_{1}$ и $\ell_{2}$. Кстати, на такие задачи (можно рассматривать полосу в плоском случае или трехмерную задачу с гексагональной симметрией в горизонтальных плоскостях) все предыдущие рассмотрения переносятся без каких-либо изменений. В данном случае в пространстве полей $(\boldsymbol{v}, P, \theta)$ подпространство всевозможных полей вида $(0, P(z), \theta(z))$ инвариантно. Поэтому движение (с большой точностью) будет продолжаться вдоль этого инвариантного многообразия. При этом скорость $\boldsymbol{v}=0$, давление $P$ “следит" за температурой $\theta(z, t)$ и определяется уравнением $0=-\nabla P+\theta \boldsymbol{k}$, а температура определяется как решение начально-краевой задачи для уравнения теплопроводности $(\delta>0)$ :

$$
\frac{\partial \theta}{\partial t}=\delta \frac{\partial^{2} \theta}{\partial z^{2}},\left.\quad \theta\right|_{t=0}=\theta_{+}(z),\left.\quad \theta\right|_{z=\mp h}=-z .
$$


Далее, независимо от начального значения $\theta_{+}(z)$ решение $\theta=\theta(z, t)$ при $t \rightarrow+\infty$ стремится к профилю $\theta_{0}=-z$ в самом сильном смысле (равномерно, со всеми производными). Этим заканчивается второй, медленный этап процесса, происходящий за время порядка $1 / \delta$ при $\delta \rightarrow 0$. В результате вырабатывается стандартный равновесный профиль температуры $\theta_{0}=-z$. Если бы $\boldsymbol{v}$ точно обращалась в нуль и, соответственно, температура $\theta$ в точности определялась бы системой (6.1), то на этом бы эволюция и закончилась. Однако равновесие $\theta_{0}=-z$ при больших $r$ заведомо неустойчиво, и малые возмущения приведут поэтому к новому конвективному перевороту, который начинается вблизи этого равновесия. Дальнейшая эволюция состоит в чередовании быстрых конвективных переворотов с долгими промежутками времени, когда жидкость почти не двигается, а теплопроводность перестраивает профиль температуры. Длительность этих медленных этапов может быть (в пределе $t \rightarrow+\infty$ ) постоянной (периодический режим), периодической (двухчастотный периодический режим), а также и хаотической (турбулентность).

Видимо, в приложениях нужно будет отождествить конвективный переворот с тем или иным катастрофическим явлением - таким, как извержение вулкана, землетрясение или вспышкой вспыхивающей звезды [37]. Долгие промежутки времени между последовательными конвективными переворотами при этом отождествляются со спокойными временами между катастрофами.

Заметим еще, что в магнитной гидродинамике, в проблеме магнитного динамо (см., например, [38]) при малых магнитных вязкостях ситуация вполне аналогична рассмотренной выше, так что представляется актуальным построение теории магнитных переворотов.

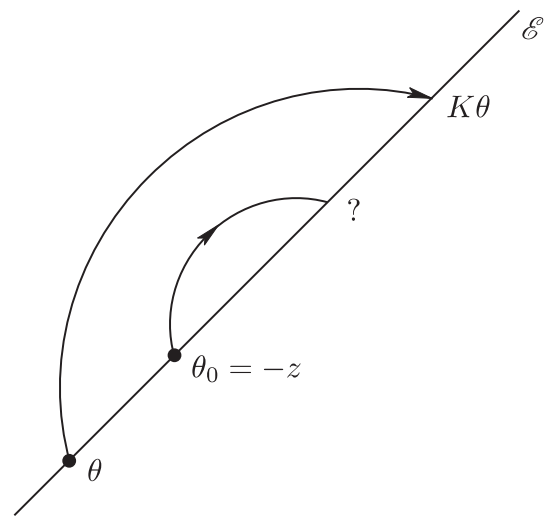

Рис. 2

В работе фактически доказано, что всякое движение начинается (при $t \rightarrow-\infty)$ и заканчивается (при $t \rightarrow+\infty)$ на подпространстве $\mathscr{E}$. Тем самым определяется отображение $K: \mathscr{E} \rightarrow \mathscr{E}$ (очевидно, не взаимно однозначное и, по-видимому, многозначное), см. рис. 2. Его свойства пока мало изучены.

Поскольку конвективный переворот начинается с $\theta_{0}=-z, \boldsymbol{v}_{0}=0$, а заканчивается в точке фазового пространства $\theta_{0}=K \theta_{0}, \boldsymbol{v}_{0}=0$, особенно интересно 
узнать, что собой представляет $K \theta_{0}$. Возникает гипотеза, что $K \theta_{0}$ есть равноизмеримая с $\theta_{0}$ и притом монотонно возрастающая функция от $z$. В случае слоя $-h / 2 \leqslant z \leqslant h / 2$ выходит, что $\left(K \theta_{0}\right)(z)=z$. Это условное утверждение было проверено обширными вычислениями [39], [40] - и полностью подтвердилось; строгим доказательством мы, однако, не располагаем.

Конечная вязкость. Почти все сложности в проблеме конвекции, включая турбулентность, вызваны теплопроводностью, которая разрушает термодинамическую замкнутость системы. Можно довольно уверенно предполагать, что в случае конечной вязкости $\mu$ основные качественные выводы о конвекции не изменятся. Это, в частности, относится к главному выводу об установлении равновесия при $t \rightarrow+\infty$ для каждого движения (разумеется, при $\mu>0$ начально-краевая задача для $t<0$ становится некорректной).

Действительно, если положить в (1.6) $\delta=0$, а уравнения (1.7) и (1.8) оставить неизменными, то из полученной системы обычным путем получаем соотношение

$$
\frac{d}{d t} \int_{D}\left(\frac{\boldsymbol{v}^{2}}{2}+\frac{\varphi^{2}}{2}\right) d x=-\mu \int_{D}(\nabla \boldsymbol{v})^{2} d x, \quad \varphi=\theta-z .
$$

Из него можно вывести, что, по-прежнему, движение жидкости затухает и устанавливается равновесие.

Обоснования, однако, существенно усложняются. Прежде всего, не удается доказать глобальную теорему существования и единственности начальнокраевой задачи в 3-мерном случае; в 2-мерном это возможно. Заметим, что система Навье-Стокса возникает как частный случай данной системы в случаe, когда $\theta(x, 0)=$ const; тогда при $\delta=0$ для всех $t>0$ также $\theta(x, t)=$ const.

В общем, случаю $\mu>0, \delta=0$ должна быть посвящена отдельная работа. Заметим лишь, что имеет место важная формула

$$
E\left[\boldsymbol{v}_{0}, f\right]-\lim _{t \rightarrow+\infty} E[\boldsymbol{v}(t), \theta(t)]=\mu \int_{0}^{\infty} \int_{D}(\nabla \boldsymbol{v})^{2} d x d t,
$$

где $E$ - полная механическая энергия жидкости:

$$
E[\boldsymbol{v}, \theta]=\int_{D}\left[\frac{\boldsymbol{v}^{2}}{2}+\frac{\varphi^{2}}{2}\right] d x d t
$$

В приложениях левую (или правую) части формулы (6.3) следует сопоставлять с энергией, выделяющейся в результате катастрофы. Слева можно положить $\boldsymbol{v}=0$.

Общие законы трения. В предыдущих рассмотрениях ньютоновский закон трения $F_{\mathrm{fr}}=\mu \Delta \boldsymbol{v}$ вовсе не обязателен. По крайней мере, на формальном уровне, с точностью до теорем существования и т.п., основной результат об установлении равновесия остается в силе и для любого закона рэлеевского трения. Иными словами, вместо оператора Лапласа может стоять $-A \boldsymbol{v}$ с произвольным положительным оператором $A$. В частности, в теории фильтрационной конвекции [41] используется закон Дарси: $F_{\text {fr }}=-k \boldsymbol{v}$, так что $A=k I$, 
$k>0$. В приложениях коэффициент трения Дарси $k$ очень велик, так что, как правило, рассматривается лишь предельный случай $k \rightarrow+\infty$.

\section{Список литературы}

[1] В.И. Юдович, "Нестационарные течения идеальной несжимаемой жидкости", ЖВМ и МФ, 3:6 (1963), 1032-1036; англ. пер.: V. I. Yudovich, "Non-stationary flow of an ideal incompressible liquid", Comput. Math. Math. Phys., 3 (1963), 1407-1456.

[2] В. И. Юдович, "О некоторых оценках, связанных с интегральными операторами и решениями эллиптических уравнений”, Докл. АН СССР, 138:4 (1961), 805808; англ. пер.: V. I. Yudovich, "Some estimates connected with integral operators and with solutions of elliptic equations", Sov. Math. Dokl., 2 (1961), 746-749.

[3] С. И. Похожаев, "О теореме вложения Соболева в случае $p l=n$ ", Доклады научно-технической конферениии Московского энергетического института, МЭИ, М., 1965, 158-170.

[4] В.И. Юдович, О конвекиии в силъно вязкой жидкости. II: Ползущие течения нетеплопроводной жидкости, Деп. в ВИНИТИ, 14.10.1992, № 2964-92.

[5] В.И. Юдович, Об уравнениях свободной конвекиии в приближении ОбербекаБуссинеска, Деп. в ВИНИТИ, 13.12.1990, № 6225-90.

[6] Г.З. Гершуни, Е. М. Жуховицкий, Конвективная устойчивость несжимаемой жидкости, Наука, М., 1972.

[7] И. И. Ворович, В. И. Юдович, "Стационарное течение вязкой несжимаемой жидкости", Матем. сб., 53(95):4 (1961), 393-428.

[8] О.А. Ладыженская, Математические вопросы динамики вязкой несэимаемой жидкости, Физматгиз, М., 1961; англ. пер.: О. A. Ladyzhenskaya, The mathematical theory of viscous incompressible flow, Gordon and Breach, New York, 1963.

[9] В. И. Юдович, Метод линеаризации в гидродинамической теории устойчивости, Изд-во РГУ, Ростов-на-Дону, 1984; англ. пер.: V.I. Yudovich, The linearization method in hydrodynamical stability theory, Transl. Math. Monogr., 74, Amer. Math. Soc., Providence, RI, 1989.

[10] V.I. Arnol'd, "Reversible systems", Nonlinear and turbulent processes in physics, vol. 3 (Kiev, 1983), Harwood Acad. Publ., Chur, 1984, 1161-1174; рус. пер.: В. И. Арнольд, "Обратимые системы", Проблемы нелинейных и турбулентных процессов в физике, II, ред. А. С. Давыдов, В. М. Черноусенко, Наукова думка, Киев, 1985, $15-21$.

[11] V.I. Arnol'd, M. B. Sevryuk, "Oscillations and bifurcations in reversible systems", Nonlinear phenomena in plasma physics and hydrodynamics, ed. R. Z. Sagdeev, Mir, Moscow, 1986, 31-64.

[12] В. И. Арнольд, “Теория катастроф”, Итоги науки и техники. Соврем. проблемъ матем. Фундам. напр. Динамические системь-5, 5, ВИНИТИ, М., 1986, 219-277; англ. пер.: V. I. Arnol'd, "Catastrophe theory", Encyclopaedia Math. Sci., Dynamical systems $V, 1994,207-264$.

[13] М. Б. Севрюк, "Бифуркации в обратимых системах", УМН, 40:5 (1985), 235-236.

[14] M. B. Sevryuk, Reversible systems, Lecture Notes in Math., 1211, Springer-Verlag, Berlin, 1986.

[15] Г. Г. Харди, Д. Е. Литтлвуд, Г. Полиа, Неравенства, ИЛ, М., 1948; пер. с англ.: G. H. Hardy, J. E. Littlewood, G. Pólya, Inequalities, Cambridge Univ. Press, Cambridge, 1934 .

[16] С. Сакс, Теория интеграла, ИЛ, М., 1949; пер. с англ.: S. Saks, Theory of the integral, Stechert, New York, 1937. 
[17] И. Стейн, Сингулярнъе интеграль и дифференциальные свойства функций, Мир, M., 1973; пер. с англ.: Е. M. Stein, Singular integrals and differentiability properties of functions, Princeton Univ. Press, Princeton, 1970.

[18] И. Стейн, Г. Вейс, Введение в гармонический анализ на евклидовых пространствах, Мир, М., 1974; пер. с англ.: Е. M. Stein, G. Weiss, Introduction to Fourier analysis on Euclidean spaces, Princeton Math. Ser., 32, Princeton Univ. Press, Princeton, 1971.

[19] П. Халмош, Лекиии по эргодической теории, ИЛ, М., 1959; пер. с англ.: P. R. Halmos, Lectures on ergodic theory, Math. Soc. Japan, 1956.

[20] П. Халмош, Теория меры, ИЛ, М., 1953; пер. с англ.: Р. R. Halmos, Measure theory, D. Van Nostrand, New York, 1950.

[21] J. V. Ryff, "Measure preserving transformations and rearrrangements", J. Math. Anal. Appl., 31 (1970), 449-458.

[22] В.И. Юдович, "Линейная устойчивость равновесий нетеплопроводной жидкости", Матем. сб., 185:5 (1994), 139-159; англ. пер.: V. I. Yudovich, "Linear stability of equilibria of a fluid that is a nonconductor of heat", Russian Acad. Sci. Sb. Math., 82:1 (1995), 117-134.

[23] R. J. Di Perna, P. L. Lions, "Ordinary differential equations, transport theory and Sobolev spaces", Invent. Math., 98 (1989), 511-547.

[24] Н. М. Гюнтер, "Об основной задаче гидродинамики", Изв. физ.-матем. ин-та им. В. А. Стеклова, 2 (1926), 1-168; "О движении жидкости, заключенной в данном перемещающемся сосуде”, Изв. АН СССР. Отд. физ.-матем. наук, 1926, 1323-1348, 1503-1532; 1927, 621-656, 735-756, 1139-1162; 1928, 9-30.

[25] L. Lichtenstein, Grundlagen der Hydromechanik, Springer-Verlag, Berlin, 1929.

[26] Ф. Хартман, Обыкновенные дифференииальные уравнения, Мир, М., 1970; пер. с англ.: Ph. Hartman, Ordinary differential equations, Wiley, New York, 1964.

[27] V.I. Yudovich, "Uniqueness theorem for the basic nonstationary problem in the dynamics of an ideal incompressible fluid", Math. Res. Lett., 2 (1995), 27-38.

[28] V. I. Yudovich, "Topics in an ideal fluid dynamics", J. Math. Fluid Mech., 7:3 (2005), 299-325.

[29] С.Л. Соболев, “Об одной теореме функционального анализа”, Матем. сб., 4:3 (1938), 471-497; англ. пер.: S. L. Sobolev, "On a theorem of functional analysis", Amer. Math. Soc. Transl. Ser. 2, 34 (1963), 39-68.

[30] С. Л. Соболев, Некоторые применения функиионального анализа в математической физике, Наука, M., 1988; англ. пер.: S. L. Sobolev, Some applications of functional analysis in mathematical physics, Amer. Math. Soc., Providence, RI, 1991.

[31] Р. Темам, Уравнения Навъе-Стокса, Мир, М., 1981; пер. с англ.: R. Tетат, Navier-Stokes equations. Theory and numerical analysis, Stud. Math. Appl., 2, NorthHolland, Amsterdam, 1977.

[32] J. Leray, "Étude de diverses équations intégrales non linéaires et de quelques problèmes que posent l'hydrodynamique", J. Math. Pures Appl., 12 (1933), 1-82.

[33] Э. Б. Быховский, Н. В. Смирнов, “Об ортогональных разложениях пространства вектор-функций, квадратично суммируемых по заданной области", Математические вопросы гидродинамики и магнитной гидродинамики для вязкой несжимаемой жидкости, Тр. МИАН, 59, Изд-во АН СССР, М., Л., 1960, 6-36.

[34] K. O. Friedrichs, "Differential forms on Riemannian manifolds", Comm. Pure Appl. Math., 8 (1955), 551-590.

[35] С. Г. Михлин, Линейные уравнения в частных производных, Высшая школа, М., 1977.

[36] В. И. Юдович, Асимптотика пределъных ииклов системы Лоренца при больших числах Релея, Деп. в ВИНИТИ, 31.07.78, 2611-78. 
[37] Г. А. Гурзадян, Вспыхивающие звезды, Наука, М., 1973; англ. пер.: G. A. Gurzadian, Flare stars, Pergamon Press, Oxford, 1980.

[38] V.I. Arnold, B. A. Khesin, Topological methods in hydrodynamics, Appl. Math. Sci., 125, Springer-Verlag, New York, 1998.

[39] Н. В. Петровская, В. И. Юдович, Компъютерный эксперимент по свободной конвекиии в слабо теплопроводной жидкости, Деп. в ВИНИТИ, № 2879-В93.

[40] М. Ю. Жуков, Н.В. Петровская, Е.В. Ширяева, Компьютерный эксперимент по свободной конвекиии в сильно вязкой, слабо теплопроводной жидкости, I, Деп. в ВИНИТИ, 11.03.2004, № 422-В2004.

[41] Д. Джозеф, Устойчивость движения жидкости, Мир, М., 1981; пер. с англ.: D. D. Joseph, Stability of fluid motions, Springer Tracts Nat. Phylos., 27, 28, Springer-Verlag, Berlin, 1976.

\section{В. И. Юдович (V. I. Yudovich)}

Поступила в редакцию

Ростовский государственный университет,

г. Ростов-на-Дону 